\title{
Modular functors are determined by their genus zero data
}

\author{
Jørgen Ellegaard Andersen and Kenji Ueno ${ }^{1}$
}

\begin{abstract}
We prove in this paper that the genus zero data of a modular functor determines the modular functor. We do this by establishing that the $S$-matrix in genus one with one point labeled arbitrarily can be expressed in terms of the genus zero information and we give an explicit formula. We do not assume the modular functor in question has duality or is unitary, in order to establish this.
\end{abstract}

Mathematics Subject Classification (2010). 57R56.

Keywords. Topological quantum field theory, modular functors, curve operators, mapping class groups.

\section{Contents}

1 Introduction . . . . . . . . . . . . . . . . 255

2 The axioms for a modular functor . . . . . . . . . . . . . . . 260

3 Basic data . . . . . . . . . . . . . . . . 266

4 Curve operators . . . . . . . . . . . . . . . . . . 272

5 The relation between curve operators and Dehn-twists . . . . . . . . . 274

6 The reduction from a once punctured genus one surfaces to genus zero . . 275

7 Consequences of the universal relations on the basic data $\ldots \ldots$. . . . 279

8 The formula for $S(\lambda) \ldots \ldots \ldots \ldots \ldots \ldots$

References . . . . . . . . . . . . . . . . . . . . . 289

\section{Introduction}

A modular functor is a functor $Z$ from the category of smooth surfaces with certain extra structure, namely the category of labeled marked surfaces, to the category of finite-dimensional vector spaces over the complex numbers. In Section 2, we shall

\footnotetext{
${ }^{1}$ This article was partially supported by the center of excellence grant "Center for Quantum Geometry of Moduli Spaces" from the Danish National Research Foundation and by Grant-in-Aid for Scientific Research No. 19340007 from JSPS (Japan Society for the Promotion of Science).
} 
give the precise axioms for a modular functor following K. Walker's topological reformulation [34] of G. Segal's axioms for a conformal field theory [28].

The objects of the category of labeled marked surfaces are pairs consisting of a marked surface and a labeling. A marked surface here refers to a quadruple of structures: a smooth closed oriented surface, a finite set of "marked" points, a tangent direction at each of the marked points together with a Lagrangian subspace of the first homology of the surface. Labeled means that each marked point of the surface is labeled by an element from a certain finite label-set $\Lambda$ which is specific to the modular functor $Z$. This label set is further required to have an involution ${ }^{\dagger}$ and a preferred $0 \in \Lambda$, such that $0^{\dagger}=0$. By the factorization property of a modular functor, we can express the vector space associated to any label marked surface as a direct sum of tensor products of vector spaces associated to 2-spheres with three marked points labeled appropriately (see Section 3 for further details). This is done by choosing a pair of pants decomposition of the surface. Let $S^{2}=\mathbb{C} \cap\{\infty\}$ and $v_{t}$ be the direction along the positive real axis at $t \in \mathbf{R} \cup \infty \subset S^{2}$. We let $\Upsilon=\left(S^{2} ; 0,1, \infty ; v_{0}, v_{1}, v_{\infty}\right)$ as illustrated in Figure 1. Note that, for simplicity, we do not illustrate the tangent vectors on any of our figures. For $\lambda, \mu, v \in \Lambda$, we define

$$
Z_{\lambda, \mu, \nu}=Z(\Upsilon, \lambda, \mu, \nu)
$$

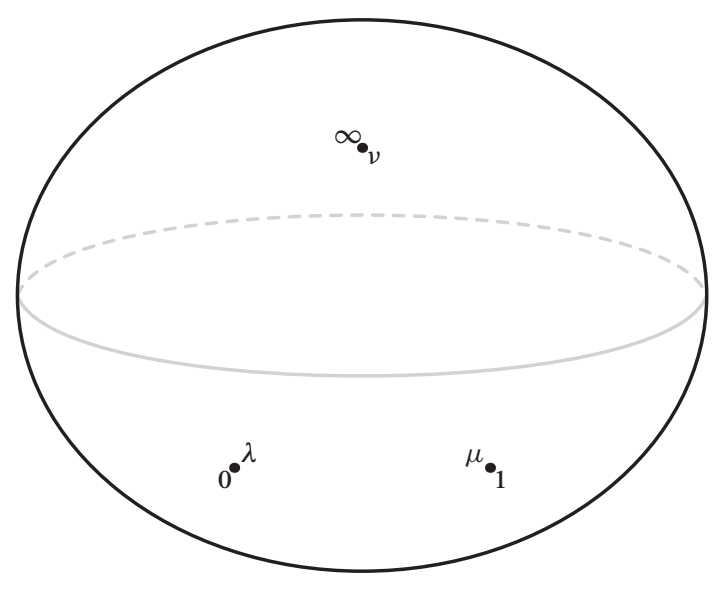

Figure 1. A sphere with three labeled marked points.

Any two pair of pants decompositions of a given surface can be obtained, one from the other by a sequence of flips. Two kinds of flips are needed. The first one is the change from the pair of pants decomposition of the 2-sphere with four marked points given by $\gamma_{1}$ to the one given by $\gamma_{2}$ in Figure 2. In Figure 2 we have indicated labels next to the two curves on the surface. These indicate the corresponding labels after factorization in the respective curves. We will use this convention throughout 


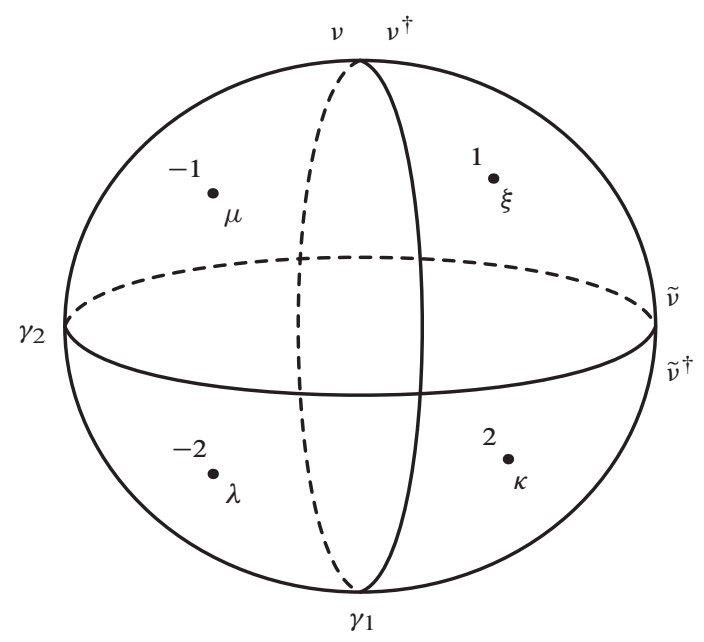

Figure 2. Two pair of pants decompositions of a sphere with four labeled marked points.

the paper. As will be explained in Section 3, these two different factorizations of the same surface gives us an isomorphism

$$
F\left[\begin{array}{cc}
\mu & \xi \\
\lambda & \kappa
\end{array}\right]: \bigoplus_{\nu \in \Lambda} Z_{v, \mu, \lambda} \otimes Z_{v^{\dagger}, \kappa, \xi} \longrightarrow \bigoplus_{\tilde{v} \in \Lambda} Z_{\tilde{v}, \lambda, \kappa} \otimes Z_{\tilde{v}^{\dagger}, \xi, \mu}
$$

The second kind of flip is the change from the pair of pants decomposition of the torus with one marked point given by $\alpha$ to the one given by $\beta$ in Figure 3. For each label $\lambda \in \Lambda$, we get an isomorphism

$$
S(\lambda): \bigoplus_{\mu} Z_{\lambda, \mu, \mu^{\dagger}} \longrightarrow \bigoplus_{\nu} Z_{\lambda, \nu, v^{\dagger}}
$$

For $\lambda=0$, we simply write $S=S(0)$. From the axioms of a modular functor, it follows that $\operatorname{dim} Z_{0, \mu, \mu^{\dagger}}=1$ for all $\mu \in \Lambda$. Further, we will see in Section 3 that the axioms of a modular functor determine a unique non-zero vector in $Z_{0, \mu, \mu^{\dagger}}$, and so we get a matrix $S=\left(S_{\mu, v}\right)_{\mu, \nu \in \Lambda}$. A flip relating two pair of pants decompositions gives an isomorphism between the corresponding two direct sums of tensor products of the vector spaces $Z_{\lambda, \mu, v}$ for appropriately varying $\lambda, \mu, v \in \Lambda$. For the first flip, the corresponding isomorphism is clearly determined by the functor applied to genus zero surfaces with less than or equal to four marked points. On the other hand, the second flip involves a genus one surface with one marked point. However, we will prove in this paper that $S(\lambda)$ for all $\lambda \in \Lambda$ is determined by the restriction of the modular functor to genus zero surfaces. We call this restriction to genus zero surfaces of a modular functor the genus zero data of the modular functor. 


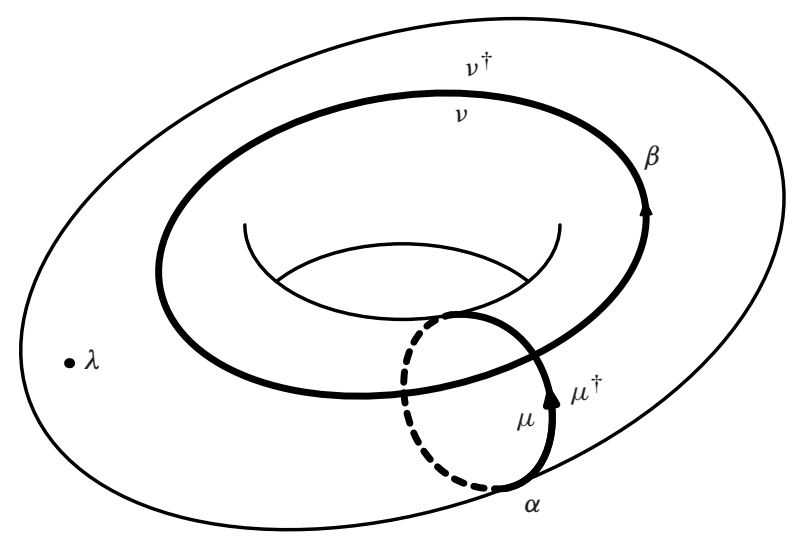

Figure 3. Two pair of pants decompositions of a torus with one labeled marked point.

Theorem 1.1. The genus zero data of any modular functor determines the modular functor.

We stress that we do not assume that our modular functor satisfies neither the orientation reversal axiom (Axiom MF-D, Section 2) nor the unitarity axiom (Axiom MF-U, Section 2) in order to prove this theorem; hence, it applies to modular functors which are not known to admit either duality structure or a unitarity structure. Please see Section 2 for the precise axioms of a modular functor.

The key ingredient in the proof of this theorem is what we call the curve operators. Given an oriented, simple, closed curve $\gamma$ on a labeled marked surface and a label $\lambda_{\gamma} \in \Lambda$, we construct an endomorphism of the vector space the modular functor associates to the labeled marked surface. Loosely speaking, they are obtained by creating two points labeled by $\lambda_{\gamma}$ and $\lambda_{\gamma}^{\dagger}$ near each other along $\gamma$. Move one of them around $\gamma$ and then annihilate them again. For the precise definition see Sections 4. The next step is to express the automorphism induced by a Dehn-twist in a simple closed curve as a linear combination of curve operators for the same curve. In fact this linear combination is seen to be universal by factoring along the boundary of a tubular neighborhood of the curve. Moreover, it is then clear that the linear combination is also determined by genus zero data. Now, since curve operators are determined completely by genus zero data, as we argue in Section 6, we see that the Dehn-twist in any simple closed curve is determined by genus zero data. Using the standard presentation of the mapping class group of a surface of genus one with one marked point, we conclude that the matrices $S(\lambda)$ are also determined by the genus zero data.

In fact, we will establish the following explicit formula for $S(\lambda)$. Pick a basis $\zeta_{j}(\lambda, \mu, v), j=1, \ldots \operatorname{dim} Z_{\lambda, \mu, v}$ for $Z_{\lambda, \mu, v}$. For $v=\mu^{\dagger}$ and $\lambda=0$, we will assume that $\zeta_{1}\left(0, \mu, \mu^{\dagger}\right)$ is this preferred vector in $Z_{0, \mu, \mu^{\dagger}}$, as discussed above. We 
then define

$$
F_{\nu, \tilde{v}}\left[\begin{array}{ll}
\mu & \xi \\
\lambda & \kappa
\end{array}\right]\left(\zeta_{i}(\nu, \mu, \lambda) \otimes \zeta_{j}\left(v^{\dagger}, \kappa, \xi\right)\right)=\sum_{k, l} F_{v, \tilde{v}}\left[\begin{array}{ll}
\mu & \xi \\
\lambda & \kappa
\end{array}\right]_{i j}^{k l} \zeta_{k}(\tilde{v}, \lambda, \kappa) \otimes \zeta_{l}\left(\tilde{v}^{\dagger}, \xi, \mu\right)
$$

and

$$
S(\lambda)\left(\zeta_{i}\left(\lambda, \mu, \mu^{\dagger}\right)\right)=\sum_{\nu, j} S(\lambda)_{\mu, i}^{\nu, j} \zeta_{j}\left(\lambda, \nu, \nu^{\dagger}\right)
$$

The determination of these linear maps will be discussed in more detail in Section 3. We further need the following three self-diffeomorphisms of $\Upsilon$. The diffeomorphism $R: \Upsilon \rightarrow \Upsilon$ is $R(z)=(z-1) / z$. It induces a linear isomorphism

$$
R: Z_{\lambda, \mu, v} \longrightarrow Z_{\mu, v, \lambda}
$$

with the matrix presentation

$$
R\left(\zeta_{i}(\lambda, \mu, \nu)\right)=\sum_{j} R_{i j} \zeta_{j}(\mu, v, \lambda)
$$

The diffeomorphism $B: \Upsilon \rightarrow \Upsilon$ is the composition of $z \mapsto z /(z-1)$ with a negative half-twist at 0 and $\infty$ and a positive half-twist at 1 . Again, it induces a linear isomorphism

$$
B: Z_{\lambda, \mu, v} \longrightarrow Z_{\lambda, \nu, \mu}
$$

with the matrix presentation

$$
B\left(\zeta_{i}(\lambda, \mu, \nu)\right)=\sum_{j} B_{i j} \zeta_{j}(\lambda, \nu, \mu)
$$

Finally, the Dehn twist in a circle centered in 0 and of a radius bigger than 1 induces a further endomorphism of $\Upsilon$. In particular, it induces an isomorphism of $Z_{0, \mu, \mu^{\dagger}}$ to itself. But since this space is 1-dimensional, this isomorphism is simply given by multiplication by a non-zero complex number $d_{\mu}$.

We introduce the so-called twisted F-isomorphism

$$
\tilde{F}_{\nu, \tilde{v}}\left[\begin{array}{cc}
\mu & \xi \\
\lambda & \kappa
\end{array}\right]: \bigoplus_{\nu \in \Lambda} Z_{\nu, \mu, \lambda} \otimes Z_{\xi, \nu^{\dagger}, \kappa} \longrightarrow \bigoplus_{\tilde{v} \in \Lambda} Z_{\kappa, \lambda, \tilde{v}} \otimes Z_{\tilde{v}^{\dagger}, \xi, \mu}
$$

defined by

$$
\widetilde{F}_{\nu, \tilde{v}}\left[\begin{array}{ll}
\mu & \xi \\
\lambda & \kappa
\end{array}\right]=\left(B R^{2} \otimes \mathrm{Id}\right) F_{\nu, \tilde{v}}\left[\begin{array}{ll}
\mu & \xi \\
\lambda & \kappa
\end{array}\right](\mathrm{Id} \otimes B R) .
$$

In the matrix presentation we get

$$
\widetilde{F}_{v, \tilde{v}}\left[\begin{array}{ll}
\mu & \xi \\
\lambda & \kappa
\end{array}\right]_{k i}^{j m}=\sum_{p, r, s, w} R_{i p} B_{p r} R_{s w}^{2} B_{w j} F_{v, \tilde{v}}\left[\begin{array}{ll}
\mu & \xi \\
\lambda & \kappa
\end{array}\right]_{k r}^{s m}
$$


Theorem 1.2. For any modular functor we have the following equation:

$$
S(\lambda)_{\mu, i}^{v, j}=\varepsilon \sum_{\kappa, m, k} d_{\kappa}^{-1} d_{\mu} S_{\kappa^{\dagger}, 0} \widetilde{F}_{\mu^{\dagger}, \nu}\left[\begin{array}{cc}
\kappa & \mu^{\dagger} \\
\nu^{\dagger} & \lambda
\end{array}\right]_{k i}^{j m} R_{m k} .
$$

Here, $\varepsilon \in \mathbb{C}^{*}$ is the so-called central charge of the modular functor $Z$ as defined in Section 2 , which is of course clearly determined by the genus zero data. Theorem 1.2 alone does not completely prove that $S(\lambda)$ is determined by genus zero data, since it involves $S_{\kappa^{\dagger}, 0}$. However, we will in fact first argue that $S(\lambda)$ for all $\lambda \in \Lambda$ is determined by genus zero data, in particular $S_{\kappa^{\dagger}, 0}=S(0)_{\kappa^{\dagger}, 1}^{0,1}$.

This paper is organized as follows. We present the axioms of a modular functor in Section 2. In Section 3, we recall the notion of basic data as defined by Kevin Walker. The curve operators are introduced in Section 4. In Section 5, we establish that the Dehn-twist in any curve can be expressed as a linear combination of the curve operators associated to the curve. This rests on Proposition 5.2, which is proved in Section 7. In Section 6, it is argued that the Dehn-twist in the $\beta$ on the genus one surface in Figure 3 is determined via genus zero data with respect to the factorization along the curve $\alpha$. This combined with relations in the mapping class group of a once marked genus one surface gives a proof of Theorem 1.1. A couple of formulae involving $F$ and $S$ are derived in Section 7 followed by a proof of Proposition 5.2. In Section 8 , we derive the formula for the $S(\lambda)$-matrix.

This paper is the third paper in a series of four, the other three being [11], [12], and [13]. In the first two papers, we construct modular functors from Conformal Field Theory, and in the fourth, we identify the resulting modular functors which underlie the Reshetikhin-Turaev TQFT [26], [27], and [30] via the Skein theory realizations of Blanchet, Habegger, Masbaum and Vogel [17], [18], and [16]. In that paper we first identify the genus zero part of the two theories. We then rely on the result of this paper to extend this identification to all genera. For applications of this identification see e.g. [1], [10], [2], [3], [4], [8], [6], and [7].

\section{The axioms for a modular functor}

In this section, we shall give the axioms for a modular functor. These are due to Moore and Seiberg [24] and [25] and to G. Segal [28]. We present them here in a topological form, which is due to K. Walker [34]; see also [21]. We note that similar, but different, axioms for a modular functor are given in [30] and in [15]. It is, however, not clear if these definitions of a modular functor are equivalent to the one presented here.

Let us start by fixing a bit of notation. By a closed surface, we mean a smooth, real, 2-dimensional manifold. For a closed oriented surface $\Sigma$ of genus $g$, we have the non-degenerate skew-symmetric intersection pairing

$$
(\cdot, \cdot): H_{1}(\Sigma, \mathbb{Z}) \times H_{1}(\Sigma, \mathbb{Z}) \longrightarrow \mathbb{Z} .
$$


Suppose $\Sigma$ is connected. In this case, a Lagrangian subspace $L \subset H_{1}(\Sigma, \mathbb{Z})$ is by definition a subspace, which is maximally isotropic with respect to the intersection pairing. A $\mathbb{Z}$-basis $(\vec{\alpha}, \vec{\beta})=\left(\alpha_{1}, \ldots, \alpha_{g}, \beta_{1}, \ldots \beta_{g}\right)$ for $H_{1}(\Sigma, \mathbb{Z})$ is called $a$ symplectic basis if

$$
\left(\alpha_{i}, \beta_{j}\right)=\delta_{i j}, \quad \text { and } \quad\left(\alpha_{i}, \alpha_{j}\right)=\left(\beta_{i}, \beta_{j}\right)=0,
$$

for all $i, j=1, \ldots, g$.

If $\Sigma$ is not connected, then $H_{1}(\Sigma, \mathbb{Z})=\bigoplus_{i} H_{1}\left(\Sigma_{i}, \mathbb{Z}\right)$, where $\Sigma_{i}$ are the connected components of $\Sigma$. In this paper we define a Lagrangian subspace of $H_{1}(\Sigma, \mathbb{Z})$ to be a subspace of the form $L=\bigoplus_{i} L_{i}$, where $L_{i} \subset H_{1}\left(\Sigma_{i}, \mathbb{Z}\right)$ is Lagrangian. Likewise, a symplectic basis for $H_{1}(\Sigma, \mathbb{Z})$ is a $\mathbb{Z}$-basis of the form $\left(\left(\vec{\alpha}^{i}, \vec{\beta}^{i}\right)\right)$, where $\left(\vec{\alpha}^{i}, \vec{\beta}^{i}\right)$ is a symplectic basis for $H_{1}\left(\Sigma_{i}, \mathbb{Z}\right)$.

For any real vector space $V$, we define $P V=(V-\{0\}) / \mathbb{R}+$.

Definition 2.1. A pointed surface $(\Sigma, P)$ is an oriented closed surface $\Sigma$ with a finite set $P \subset \Sigma$ of points. A pointed surface is called stable if the Euler characteristic of each component of the complement of the points $P$ is negative. A pointed surface is called saturated if each component of $\Sigma$ contains at least one point from $P$.

Definition 2.2. A morphism of pointed surfaces $f:\left(\Sigma_{1}, P_{1}\right) \rightarrow\left(\Sigma_{2}, P_{2}\right)$ is an isotopy class of orientation preserving diffeomorphisms which maps $P_{1}$ to $P_{2}$. Here the isotopy is required not to change the induced map of the first order Jet at $P_{1}$ to the first order Jet at $P_{2}$.

Definition 2.3. A marked surface $\Sigma=(\Sigma, P, V, L)$ is an oriented closed smooth surface $\Sigma$ with a finite subset $P \subset \Sigma$ of points with projective tangent vectors $V \in \sqcup_{p \in P} P T_{p} \Sigma$ and a Lagrangian subspace $L \subset H_{1}(\Sigma, \mathbb{Z})$.

The notions of stable and saturated marked surfaces are defined just like those of pointed surfaces.

In the case of genus zero, we omit the Lagrangian subspace from the discussion since, in this case, it can only be zero subspace.

Definition 2.4. A morphism $\mathbf{f}: \boldsymbol{\Sigma}_{1} \rightarrow \boldsymbol{\Sigma}_{2}$ of marked surfaces $\boldsymbol{\Sigma}_{i}=\left(\Sigma_{i}, P_{i}, V_{i}, L_{i}\right)$ is an isotopy class of orientation preserving diffeomorphisms $f: \Sigma_{1} \rightarrow \Sigma_{2}$ that maps $\left(P_{1}, V_{1}\right)$ to $\left(P_{2}, V_{2}\right)$ together with an integer $s$. Hence we write $\mathbf{f}=(f, s)$.

Remark 2.5. Any marked surface has an underlying pointed surface, but a morphism of marked surfaces does not quite induce a morphism of pointed surfaces, since we only require that the isotopies preserve the induced maps on the projective tangent spaces.

Remark 2.6. If in the notation above, we only specify $f: \Sigma_{1} \rightarrow \Sigma_{2}$, then it is assumed that the integer $s=0$. 
Let $\sigma$ be Wall's signature cocycle for triples of Lagrangian subspaces of $H_{1}(\Sigma, \mathbb{R})$; see [35].

Definition 2.7. Let $\mathbf{f}_{1}=\left(f_{1}, s_{1}\right): \boldsymbol{\Sigma}_{1} \rightarrow \boldsymbol{\Sigma}_{2}$ and $\mathbf{f}_{2}=\left(f_{2}, s_{2}\right): \boldsymbol{\Sigma}_{2} \rightarrow \boldsymbol{\Sigma}_{3}$ be morphisms of marked surfaces $\boldsymbol{\Sigma}_{i}=\left(\Sigma_{i}, P_{i}, V_{i}, L_{i}\right)$ then the composition of $\mathbf{f}_{1}$ and $\mathbf{f}_{2}$ is

$$
\mathbf{f}_{2} \mathbf{f}_{1}=\left(f_{2} f_{1}, s_{2}+s_{1}-\sigma\left(\left(f_{2} f_{1}\right)_{*} L_{1}, f_{2 *} L_{2}, L_{3}\right)\right) .
$$

With the objects being marked surfaces and the morphism and their composition being defined as in the above definition, we have constructed the category of marked surfaces.

The mapping class group $\Gamma(\Sigma)$ of a marked surface $\Sigma=(\Sigma, L)$ is the group of automorphisms of $\boldsymbol{\Sigma}$. One can prove that $\Gamma(\boldsymbol{\Sigma})$ is a central extension of the mapping class group $\Gamma(\Sigma)$ of the surface $\Sigma$ defined by the 2-cocycle $c: \Gamma(\Sigma) \rightarrow$ $\mathbb{Z}, c\left(f_{1}, f_{2}\right)=\sigma\left(\left(f_{1} f_{2}\right)_{*} L, f_{1 *} L, L\right)$. One can also prove that this cocycle is equivalent to the cocycle obtained by considering two-framings on mapping cylinders; see [14] and [5].

Notice also that for any morphism $(f, s): \Sigma_{1} \rightarrow \Sigma_{2}$, one can factor

$$
\begin{aligned}
(f, s) & =\left(\left(\mathrm{Id}, s^{\prime}\right): \boldsymbol{\Sigma}_{2} \rightarrow \boldsymbol{\Sigma}_{2}\right) \circ\left(f, s-s^{\prime}\right) \\
& =\left(f, s-s^{\prime}\right) \circ\left(\left(\mathrm{Id}, s^{\prime}\right): \boldsymbol{\Sigma}_{1} \rightarrow \boldsymbol{\Sigma}_{1}\right) .
\end{aligned}
$$

In particular $(\operatorname{Id}, s): \boldsymbol{\Sigma} \rightarrow \boldsymbol{\Sigma}$ is $(\operatorname{Id}, 1)^{s}$.

Definition 2.8. The operation of disjoint union of marked surfaces is

$$
\left(\Sigma_{1}, P_{1}, V_{1}, L_{1}\right) \sqcup\left(\Sigma_{2}, P_{2}, V_{2}, L_{2}\right)=\left(\Sigma_{1} \sqcup \Sigma_{2}, P_{1} \sqcup P_{2}, V_{1} \sqcup V_{2}, L_{1} \oplus L_{2}\right) .
$$

Morphisms on disjoint unions are accordingly

$$
\left(f_{1}, s_{1}\right) \sqcup\left(f_{2}, s_{2}\right)=\left(f_{1} \sqcup f_{2}, s_{1}+s_{2}\right) .
$$

We see that disjoint union is an operation on the category of marked surfaces.

Definition 2.9. Let $\boldsymbol{\Sigma}$ be a marked surface. We denote by $-\boldsymbol{\Sigma}$ the marked surface obtained from $\Sigma$ by the operation of reversal of the orientation. For a morphism $\mathbf{f}=(f, s): \Sigma_{1} \rightarrow \Sigma_{2}$ we let the orientation reversed morphism be given by $-\mathbf{f}=$ $(f,-s):-\Sigma_{1} \rightarrow-\Sigma_{2}$.

We also see that orientation reversal is an operation on the category of marked surfaces. Let us now consider gluing of marked surfaces.

Let $\left(\Sigma,\left\{p_{-}, p_{+}\right\} \sqcup P,\left\{v_{-}, v_{+}\right\} \sqcup V, L\right)$ be a marked surface, where we have selected an ordered pair of marked points with projective tangent vectors $\left(\left(p_{-}, v_{-}\right)\right.$, $\left.\left(p_{+}, v_{+}\right)\right)$, at which we will perform the gluing. 
Let $c: P\left(T_{p_{-}} \Sigma\right) \rightarrow P\left(T_{p_{+}} \Sigma\right)$ be an orientation reversing projective linear isomorphism such that $c\left(v_{-}\right)=v_{+}$. Such a $c$ is called a gluing map for $\Sigma$. Let $\tilde{\Sigma}$ be the oriented surface with boundary obtained from $\Sigma$ by blowing up $p_{-}$and $p_{+}$, i.e.

$$
\widetilde{\Sigma}=\left(\Sigma-\left\{p_{-}, p_{+}\right\}\right) \sqcup P\left(T_{p_{-}} \Sigma\right) \sqcup P\left(T_{p_{+}} \Sigma\right),
$$

with the natural smooth structure induced from $\Sigma$. Let now $\Sigma_{c}$ be the closed oriented surface obtained from $\widetilde{\Sigma}$ by using $c$ to glue the boundary components of $\widetilde{\Sigma}$. We call $\Sigma_{c}$ the gluing of $\Sigma$ at the ordered pair $\left(\left(p_{-}, v_{-}\right),\left(p_{+}, v_{+}\right)\right)$with respect to $c$.

Let now $\Sigma^{\prime}$ be the topological space obtained from $\Sigma$ by identifying $p_{-}$and $p_{+}$. We then have natural continuous maps $q: \Sigma_{c} \rightarrow \Sigma^{\prime}$ and $n: \Sigma \rightarrow \Sigma^{\prime}$. On the first homology group, $n$ induces an injection and $q$ a surjection, so we can define a Lagrangian subspace $L_{c} \subset H_{1}\left(\Sigma_{c}, \mathbb{Z}\right)$ by $L_{c}=q_{*}^{-1}\left(n_{*}(L)\right)$. We note that the image of $P\left(T_{p_{-}} \Sigma\right)$ (with the orientation induced from $\widetilde{\Sigma}$ ) induces naturally an element in $H_{1}\left(\Sigma_{c}, \mathbb{Z}\right)$ and as such it is contained in $L_{c}$.

Remark 2.10. If we have two gluing maps $c_{i}: P\left(T_{p_{-}} \Sigma\right) \rightarrow P\left(T_{p_{+}} \Sigma\right), i=1,2$, we note that there is a diffeomorphism $f$ of $\Sigma$ inducing the identity on $\left(p_{-}, v_{-}\right) \sqcup$ $\left(p_{+}, v_{+}\right) \sqcup(P, V)$ which is isotopic to the identity among such maps, such that $\left(d f_{p_{+}}\right)^{-1} c_{2} d f_{p_{-}}=c_{1}$. In particular, $f$ induces a diffeomorphism $f: \Sigma_{c_{1}} \rightarrow \Sigma_{c_{2}}$ compatible with $f: \Sigma \rightarrow \Sigma$, which maps $L_{c_{1}}$ to $L_{c_{2}}$. Any two such diffeomorphims of $\Sigma$ induce isotopic diffeomorphims from $\Sigma_{1}$ to $\Sigma_{2}$.

Definition 2.11. Let $\Sigma=\left(\Sigma,\left\{p_{-}, p_{+}\right\} \sqcup P,\left\{v_{-}, v_{+}\right\} \sqcup V, L\right)$ be a marked surface. Let

$$
c: P\left(T_{p_{-}} \Sigma\right) \longrightarrow P\left(T_{p_{+}} \Sigma\right)
$$

be a gluing map and $\Sigma_{c}$ the gluing of $\Sigma$ at the ordered pair $\left(\left(p_{-}, v_{-}\right),\left(p_{+}, v_{+}\right)\right)$with respect to $c$. Let $L_{c} \subset H_{1}\left(\Sigma_{c}, \mathbb{Z}\right)$ be the Lagrangian subspace constructed above from $L$. Then the marked surface $\Sigma_{c}=\left(\Sigma_{c}, P, V, L_{c}\right)$ is defined to be the gluing of $\boldsymbol{\Sigma}$ at the ordered pair $\left(\left(p_{-}, v_{-}\right),\left(p_{+}, v_{+}\right)\right)$with respect to $c$.

We observe that gluing also extends to morphisms of marked surfaces which preserve the ordered pair $\left(\left(p_{-}, v_{-}\right),\left(p_{+}, v_{+}\right)\right)$, by using gluing maps which are compatible with the morphism in question.

Remark 2.12. Let $\Sigma=(\Sigma, P, V, L)$ be marked surface. Assume that $\gamma$ is an oriented, simple, closed curve on $\Sigma-P$, such that $[\gamma] \in L$. Assume further we have a point $p$ on $\gamma$. We can then cut $\Sigma$ along $\gamma$ and obtain a surface with two boundary components, which are naturally identified with $\gamma$. By identifying each of the two boundary components to a point, say $\left\{p^{\prime}, p^{\prime \prime}\right\}$, we get a new closed surface $\widetilde{\Sigma}$, with a set of marked points $\widetilde{P}=O \cup\left\{p^{\prime}, p^{\prime \prime}\right\}$ and tangent vectors $\widetilde{V}=V \cup\left\{v^{\prime}, v^{\prime \prime}\right\}$. Here $v^{\prime}$ and $v^{\prime \prime}$ are induced by $p \in \gamma$ under the natural identification of $\gamma$ with $P\left(T_{p_{-}} \Sigma\right)$ and with $P\left(T_{p_{+}} \Sigma\right)$. Let $\Sigma^{\prime}$ be obtained from $\widetilde{\Sigma}$ by identifying $p^{\prime}$ with $p^{\prime \prime}$. We have 
the quotient map $q: \Sigma \rightarrow \Sigma^{\prime}$ and the identification map $n: \widetilde{\Sigma} \rightarrow \Sigma^{\prime}$. We specify a Lagrangian subspace in $\widetilde{L} \subset H_{1}(\widetilde{\Sigma}, \mathbf{Z})$ by $\widetilde{L}=n_{*}^{-1} q_{*}(L)$.

We say that $\tilde{\Sigma}=(\widetilde{\Sigma}, \widetilde{P}, \widetilde{V}, \widetilde{L})$ is obtained from $\Sigma$ by factoring $\Sigma$ along $(\gamma, p)$. The operation of factoring is an inverse to gluing.

We can now give the axioms for a 2-dimensional modular functor.

Definition 2.13. A label set $\Lambda$ is a finite set furnished with an involution $\lambda \mapsto \lambda^{\dagger}$ and a trivial element 0 such that $0^{\dagger}=0$.

Definition 2.14. Let $\Lambda$ be a label set. The category of $\Lambda$-labeled marked surfaces consists of marked surfaces with an element of $\Lambda$ assigned to each of the marked points, and morphisms of labeled marked surfaces are required to preserve the labelings. An assignment of elements of $\Lambda$ to the marked points of $\boldsymbol{\Sigma}$ is called a labeling of $\Sigma$, and we denote the labeled marked surface by $(\Sigma, \lambda)$, where $\lambda$ is the labeling.

We define a labeled pointed surface similarly.

Remark 2.15. The operation of disjoint union clearly extends to labeled marked surfaces. When we extend the operation of orientation reversal to labeled marked surfaces, we also apply the involution $(\cdot)^{\dagger}$ to all the labels.

Definition 2.16. A modular functor based on the label set $\Lambda$ is a functor $V$ from the category of labeled marked surfaces to the category of finite-dimensional, complex vector spaces satisfying Axioms MF1-MF5 below.

MF1 (disjoint union axiom). The operation of disjoint union of labeled marked surfaces is taken to the operation of tensor product, i.e. for any pair of labeled marked surfaces, there is an isomorphism

$$
\left.V\left(\left(\Sigma_{1}, \lambda_{1}\right) \sqcup\left(\Sigma_{2}, \lambda_{2}\right)\right)\right) \cong V\left(\Sigma_{1}, \lambda_{1}\right) \otimes V\left(\Sigma_{2}, \lambda_{2}\right) .
$$

The identification is associative.

MF2 (gluing axiom). Let $\boldsymbol{\Sigma}$ and $\boldsymbol{\Sigma}_{c}$ be marked surfaces such that $\boldsymbol{\Sigma}_{c}$ is obtained from $\Sigma$ by gluing at an ordered pair of points and projective tangent vectors with respect to a gluing map $c$. Then there is an isomorphism

$$
V\left(\Sigma_{c}, \lambda\right) \cong \bigoplus_{\mu \in \Lambda} V\left(\Sigma, \mu, \mu^{\dagger}, \lambda\right)
$$

which is associative, compatible with gluing of morphisms, as well as disjoint unions, and it is independent of the choice of the gluing map in the obvious way (see Remark 2.10). 
MF3 (empty surface axiom). Let $\emptyset$ denote the empty labeled marked surface. Then

$$
\operatorname{dim} V(\varnothing)=1 .
$$

MF4 (once punctured sphere axiom). Let $\Sigma=\left(S^{2},\{p\},\{v\}, 0\right)$ be a marked sphere with one marked point. Then

$$
\operatorname{dim} V(\Sigma, \lambda)= \begin{cases}1, & \lambda=0, \\ 0, & \lambda \neq 0 .\end{cases}
$$

MF5 (twice punctured sphere axiom). Let $\boldsymbol{\Sigma}=\left(S^{2},\left\{p_{1}, p_{2}\right\},\left\{v_{1}, v_{2}\right\},\{0\}\right)$ be a marked sphere with two marked points. Then

$$
\operatorname{dim} V(\Sigma,(\lambda, \mu))= \begin{cases}1, & \lambda=\mu^{\dagger} \\ 0, & \lambda \neq \mu^{\dagger}\end{cases}
$$

In addition to the above axioms, one may have two extra properties.

MF-D (orientation reversal axiom). The operation of orientation reversal of labeled marked surfaces is taken to the operation of taking the dual vector space, i.e. for any labeled marked surface $(\Sigma, \lambda)$ there is a pairing

$$
\langle\cdot, \cdot\rangle: V(\Sigma, \lambda) \otimes V\left(-\Sigma, \lambda^{\dagger}\right) \rightarrow \mathbb{C},
$$

compatible with disjoint unions, gluings and orientation reversals (in the sense that the induced isomorphisms $V(\Sigma, \lambda) \cong V\left(-\Sigma, \lambda^{\dagger}\right)^{*}$ and $V\left(-\Sigma, \lambda^{\dagger}\right) \cong V(\Sigma, \lambda)^{*}$ are adjoints).

MF-U (unitarity axiom). Every vector space $V(\Sigma, \lambda)$ is furnished with a hermitian inner product

$$
(\cdot, \cdot): V(\Sigma, \lambda) \otimes \overline{V(\Sigma, \lambda)} \rightarrow \mathbb{C}
$$

so that morphisms induce unitary transformation. The hermitian structure must be compatible with disjoint union and gluing. If we have the orientation reversal property, then compatibility with the unitary structure means that we have a commutative diagram

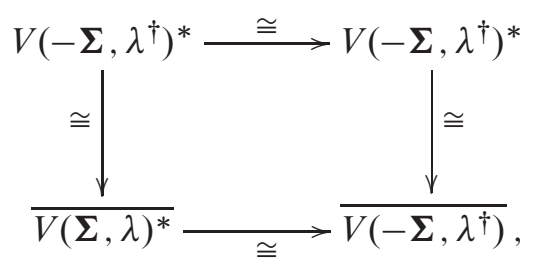


where the vertical identifications come from the hermitian structure and the horizontal ones from the duality.

Remark 2.17. By the factorization axiom combined with the disjoint union axiom, we see that, for any modular functor $Z$, there is a unique $\varepsilon \in \mathbb{C}^{*}$ such that

$$
\varepsilon \operatorname{Id}_{Z(\Sigma, \lambda)}=Z(\operatorname{Id}, 1): Z(\Sigma, \lambda) \longrightarrow Z(\Sigma, \lambda)
$$

for all labeled marked surfaces $(\Sigma, \lambda)$. In particular, $\varepsilon$ is determined by and determines the action of the mapping class group of a once pointed 0-labeled two-sphere as considered in Axiom MF4 above.

Definition 2.18. The non-zero complex scalar $\varepsilon$ with the above property is called the central change of the modular functor $Z$.

\section{Basic data}

Following Walker [34], we will review the notion of basic data. Fix throughout the rest of this paper a modular functor $Z$ with label set $(\Lambda, 0, \dagger)$. We do not assume that this modular functor $Z$ has a duality structure as in Axiom MF-D or a unitary structure as in Axiom MF-U.

We let $\Delta=\left(S^{2} ; \infty ; v_{\infty}\right), \Xi=\left(S^{2} ; 0, \infty ; v_{0}, v_{\infty}\right)$ and recall that

$$
\Upsilon=\left(S^{2} ; 0,1, \infty ; v_{0}, v_{1}, v_{\infty}\right) .
$$

For $\lambda, \mu, v \in \Lambda$ we define

$$
Z_{0}=Z(\Delta, 0), \quad Z_{\lambda, \lambda^{\dagger}}=Z\left(\Xi, \lambda, \lambda^{\dagger}\right)
$$

and recall that

$$
Z_{\lambda, \mu, \nu}=Z(\Upsilon, \lambda, \mu, \nu) .
$$

Here $\operatorname{dim} Z_{0}=\operatorname{dim} Z_{\lambda, \lambda^{\dagger}}=1$ and we define

$$
N_{\lambda, \mu}^{v}=\operatorname{dim} Z_{\lambda, \mu, \nu^{\dagger}} .
$$

The morphisms of the marked surfaces $\Delta, \Xi$ and $\Upsilon$ act on the vector spaces $Z_{0}, Z_{\lambda, \lambda^{\dagger}}$ and $Z_{\lambda, \mu, \nu}$. Recall the self morphisms $R$ and $B$ of $\Upsilon$. They, together with the Dehntwist around 0 and the morphisms (Id, 1), generate the mapping class groupoids of $\Upsilon$ with all their possible labelings. By factorization, these also determine the action of the mapping class groupoid of both $\Delta$ and $\Xi$ with all possible labelings. We shall make use of the following notation:

$$
B_{12}=R B R^{-1}, \quad B_{13}=R^{-1} B R, \quad \text { and } \quad B_{23}=B .
$$


Further, we denote by $T_{1}, T_{2}$ and $T_{3}$ the Dehn-twists around 0,1 and $\infty$, respectively.

Let $\Psi=\left(S^{2} ;-2,-1,1,2 ; v_{-2}, v_{-1}, v_{1}, v_{2}\right)$. Let $\gamma_{1}$ be the circle of radius $3 / 2$ centered in 0 and $\gamma_{2}$ the circle of radius 1 centered in $3 / 2$, both considered as simple closed curves on $\Psi$ (see Figure 2). Factoring $\Psi$ along $\left(\gamma_{1}, 3 / 2\right)$, we obtain the marked surface $\Psi_{1}$ say and likewise, factoring $\Psi$ along $\left(\gamma_{2}, 5 / 2\right)$, we obtain the marked surface $\Psi_{2}$ say. There is a unique diffeomorphism from $\Upsilon \amalg \Upsilon$ to $\Psi_{i}$ which takes the real axes to parts of the real axes on each component, infinity to the respective quotients of $\gamma_{i}$, and which maps the first copy of $\Upsilon$ to the interior of $\gamma_{i}$ in $\mathbb{C}$. We thus get two isomorphisms

$$
\Phi_{1}(\mu, \lambda): \bigoplus_{\nu \in \Lambda} Z(\Upsilon ; \nu, \mu, \lambda) \otimes Z\left(\Upsilon ; \nu^{\dagger}, \kappa, \xi\right) \longrightarrow Z(\Psi, \lambda, \mu, \xi, \kappa)
$$

and

$$
\Phi_{2}(\mu, \lambda): \bigoplus_{\tilde{v} \in \Lambda} Z(\Upsilon ; \tilde{v}, \lambda, \kappa) \otimes Z\left(\Upsilon ; \tilde{v}^{\dagger}, \xi, \mu\right) \longrightarrow Z(\Psi, \lambda, \mu, \xi, \kappa)
$$

We then define

$$
F\left[\begin{array}{c}
\mu \xi \\
\lambda \kappa
\end{array}\right]: \bigoplus_{\nu \in \Lambda} Z_{\nu, \mu, \lambda} \otimes Z_{v^{\dagger}, \kappa, \xi} \longrightarrow \bigoplus_{\tilde{v} \in \Lambda} Z_{\tilde{v}, \lambda, \kappa} \otimes Z_{\tilde{v}^{\dagger}, \xi, \mu}
$$

by

$$
F=\Phi_{2}^{-1} \circ \Phi_{1} .
$$

Let $\Theta$ be an oriented genus one surface. Let $p$ be a point on $\Theta$ and $v_{p}$ be a tangent vector at $p$. Choose two oriented, simple, closed curves $(\alpha, \beta)$ on $\Theta-\{p\}$ as indicated in Figure 3. Let $L_{\alpha}=\operatorname{Span}\{[\alpha]\}$ and $L_{\beta}=\operatorname{Span}\{[\beta]\}$ be the Lagrangian subspaces generated in the first homology group. Let

$$
\Theta^{\alpha}=\left(\Theta, p, v_{p}, L_{\alpha}\right)
$$

and

$$
\boldsymbol{\Theta}^{\beta}=\left(\Theta, p, v_{p}, L_{\beta}\right) .
$$

Let $\boldsymbol{\Theta}_{\alpha}$ and $\boldsymbol{\Theta}_{\beta}$ be marked surfaces, which result from factoring $\boldsymbol{\Theta}^{\alpha}$ along $\alpha$ and $\boldsymbol{\Theta}^{\beta}$ along $\beta$, respectively. By factorization we get isomorphisms

$$
\Phi_{\alpha}: Z\left(\Theta^{\alpha}, \lambda\right) \longrightarrow \bigoplus_{\mu} Z\left(\Theta_{\alpha}, \lambda, \mu, \mu^{\dagger}\right)
$$

and

$$
\Phi_{\beta}: Z\left(\boldsymbol{\Theta}^{\beta}, \lambda\right) \longrightarrow \bigoplus_{\nu} Z\left(\boldsymbol{\Theta}_{\beta}, \lambda, \nu, \nu^{\dagger}\right)
$$

Pick diffeomorphisms

$$
f_{\alpha}: \Upsilon \longrightarrow \boldsymbol{\Theta}_{\alpha}
$$


and

$$
f_{\beta}: \Upsilon \longrightarrow \boldsymbol{\Theta}_{\beta}
$$

which map the segment $[1, \infty]$ onto $\beta$ and $\alpha$, respectively. Then we can define

$$
S(\lambda): \bigoplus_{\mu} Z_{\lambda, \mu, \mu^{\dagger}} \longrightarrow \bigoplus_{\nu} Z_{\lambda, v, v^{\dagger}}
$$

by

$$
S(\lambda)=Z\left(f_{\beta}\right)^{-1} \Phi_{\beta} Z(\mathrm{Id}) \Phi_{\alpha}^{-1} Z\left(f_{\alpha}\right),
$$

where Id: $\left(\Theta^{\alpha}, \lambda\right) \rightarrow\left(\Theta^{\beta}, \lambda\right)$. Following Walker, eq. (3.6) in [34], we define basic data as follows:

Definition 3.1 (Walker). Basic data for the modular functor $Z$ consists of the following data.

A. The vector spaces $Z_{0}, Z_{\lambda, \lambda^{\dagger}}$ and $Z_{\lambda, \mu, \nu}$ together with the induced actions of the groupoids of morphism of marked surface acting on them.

B. The linear isomorphism

$$
F\left[\begin{array}{cc}
\mu & \xi \\
\lambda & \kappa
\end{array}\right]: \bigoplus_{\nu \in \Lambda} Z_{\nu, \mu, \lambda} \otimes Z_{v^{\dagger}, \kappa, \xi} \longrightarrow \bigoplus_{\tilde{v} \in \Lambda} Z_{\tilde{v}, \lambda, \kappa} \otimes Z_{\tilde{v}^{\dagger}, \xi, \mu}
$$

C. The linear isomorphism

$$
S(\lambda): \bigoplus_{\mu} Z_{\lambda, \mu, \mu^{\dagger}} \longrightarrow \bigoplus_{\nu} Z_{\lambda, \nu, \nu^{\dagger}}
$$

Lemma 3.2. The basic data determines the modular functor $Z$ uniquely.

This lemma is proven in Section 5 of [34]. Below, we outline the main construction behind that. Of course, A and B are, by definition, part of the genus zero data of $Z$. Further, A and B clearly determine the genus zero part of $Z$. By definition, $S(\lambda)$ requires genus one as well. But the main result of this paper is that $\mathrm{C}$ is in fact determined by $\mathrm{A}$ and $\mathrm{B}$. Hence

Theorem 3.3. The basic data under $\mathrm{A}$ and $\mathrm{B}$ determines the modular functor $Z$.

This theorem follows from Theorem 1.1, which is proven below. Let us now fix a vector $\zeta_{0} \in Z_{0}$ and $\zeta(\lambda) \in Z_{\lambda, \lambda \dagger}$. By the gluing axiom, we get natural isomorphisms

$$
Z_{0} \cong Z_{0,0} \otimes Z_{0}
$$

and

$$
Z_{\lambda, \lambda^{\dagger}} \cong Z_{\lambda, \lambda^{\dagger}} \otimes Z_{\lambda, \lambda^{\dagger}}
$$


Under these isomorphisms, we require that

$$
\zeta_{0}=\zeta(0) \otimes \zeta_{0}
$$

and

$$
\zeta(\lambda)=\zeta(\lambda) \otimes \zeta(\lambda)
$$

This condition uniquely fixes $\zeta(\lambda)$ for all $\lambda \in \Lambda$. We also need to do a computation in a basis of $Z_{\lambda, \mu, \nu}$, so we fix a basis $\zeta_{j}(\lambda, \mu, \nu), j=1, \ldots, N_{\lambda, \mu}^{v^{\dagger}}$ for each of these vector spaces. In case $N_{\lambda, \mu}^{v^{\dagger}}=1$, we use the notation $\zeta(\lambda, \mu, \nu)=\zeta_{1}(\lambda, \mu, v)$. We will require that under the isomorphism

$$
Z_{\lambda, \lambda^{\dagger}} \cong Z_{\lambda, \lambda^{\dagger}, 0} \otimes Z_{0}
$$

we have that

$$
\zeta(\lambda)=\zeta_{1}\left(\lambda, \lambda^{\dagger}, 0\right) \otimes \zeta_{0} .
$$

We write $S=S(0)$ and with respect to the preferred basis $\zeta(\lambda) \in Z_{\lambda, \lambda^{\dagger}}, \lambda \in \Lambda$, we have the matrix presentation $S=\left(S_{\lambda, \mu}\right)_{\lambda, \mu \in \Lambda}$.

Let us now recall the reconstruction of a modular functor from its basic data. On a marked surface $\Sigma$, one considers pairs $(C, \Pi)$, satisfying the following conditions.

- $C$ is a finite collection of disjoint simple closed curves, each equipped with a base point, such that the result of factoring $\Sigma$ along $C$ is a disjoint union of marked surfaces $\Sigma_{i}, i \in I$ for some finite set $I$. Further, we assume that the Lagrangian subspace of $\boldsymbol{\Sigma}$ is generated by the curves in $C$.

- $\Pi$ is a disjoint union of morphisms of marked surfaces from an ordered disjoint union of $\Delta$ 's, $\Xi$ 's and $\Upsilon$ 's to the $\boldsymbol{\Sigma}_{i}, i \in I$, covering each $\boldsymbol{\Sigma}_{i}$ exactly once.

Such a pair $(C, \Pi)$ is called an overmarking of $\Sigma$ in [34]. If $C$ is such that $|C|$ is minimal, then $(C, \Pi)$ is called a marking in [34] following [22]. We shall call these pairs decompositions so as to not confuse them with the structure of a marked surface as introduced in the previous section.

It follows from the results of [22] that any two pairs of decompositions of a given marked surface are related by a finite sequence of the following changes of decompositions from say $\left(C^{\prime}, \Pi^{\prime}\right)$ to $\left(C^{\prime \prime}, \Pi^{\prime \prime}\right)$.

$\mathcal{M}$ The collection of curves are the same $C^{\prime}=C^{\prime \prime}$, and there are automorphisms of the $\Delta$ 's, $\Xi$ 's and $\Upsilon$ 's, which relates $\Pi^{\prime}$ to $\Pi^{\prime \prime}$, namely $\left(\Pi^{\prime \prime}\right)^{-1} \Pi^{\prime}$.

$\mathcal{A}$ Insertion or removal of a component of $C^{\prime}$ to obtain $C^{\prime \prime}$ results in a corresponding insertion or removal of a copy of $\Xi$.

$D$ Insertion or removal of a component of $C^{\prime}$ to obtain $C^{\prime \prime}$ results in the replacement of one $\Xi$ by one new $\Delta$ and one new $\Upsilon$ or the converse.

$\mathscr{F}$ There are $\gamma^{\prime} \in C^{\prime}$ and $\gamma^{\prime \prime} \in C^{\prime \prime}$ with the property that $C^{\prime}-\left\{\gamma^{\prime}\right\}=C^{\prime \prime}-\left\{\gamma^{\prime \prime}\right\}$ and the factorization along $C^{\prime}-\left\{\gamma^{\prime}\right\}$ contains a component which via $\Pi^{\prime}$ and $\Pi^{\prime \prime}$ is identified with $\Psi$, such that $\gamma_{1}$ goes to $\gamma^{\prime}$ and $\gamma_{2}$ goes to $\gamma^{\prime \prime}$. 
$\delta$ There are $\gamma^{\prime} \in C^{\prime}$ and $\gamma^{\prime \prime} \in C^{\prime \prime}$ with the property that $C^{\prime}-\left\{\gamma^{\prime}\right\}=C^{\prime \prime}-\left\{\gamma^{\prime \prime}\right\}$ and the factorization along $C^{\prime}-\left\{\gamma^{\prime}\right\}$ contains a component which via $\Pi^{\prime}$ and $\Pi^{\prime \prime}$ is identified with $\left(\Theta, p, v_{p}\right)$, such that $\alpha$ goes to $\gamma^{\prime}$ and $\beta$ goes to $\gamma^{\prime \prime}$.

Let $(C, \Pi)$ be a decomposition of a labeled marked surface $(\Sigma, \lambda)$. Let $\Sigma_{C}$ be the marked surface, one obtains from factoring $\Sigma$ along $C$. Let $\Lambda_{c}=\Lambda^{\times c}$, where $c=|C|$. The factorization axiom gives us an isomorphism

$$
Z(\Sigma, \lambda) \cong \bigoplus_{\mu \in \Lambda_{C}} Z\left(\Sigma_{C}, \lambda, \mu\right)
$$

For each $\mu \in \Lambda_{c}$, we let $Z(\lambda, \mu)$ be the corresponding tensor product of the vector spaces $Z_{\lambda^{\prime}}$ 's, $Z_{\lambda^{\prime},\left(\lambda^{\prime}\right)^{\dagger}}$ 's and $Z_{\lambda^{\prime}, \mu^{\prime}, v^{\prime}}$ 's. Using $\Pi$ we then get an isomorphism

$$
Z(\Pi): Z(\lambda, \mu) \longrightarrow Z\left(\Sigma_{C}, \lambda, \mu\right)
$$

which induces an isomorphism

$$
Z(C, \Pi): \bigoplus_{\mu \in \Lambda_{c}} Z(\lambda, \mu) \longrightarrow Z(\Sigma, \lambda)
$$

Suppose now $\left(C^{\prime}, \Pi^{\prime}\right)$ and $\left(C^{\prime \prime}, \Pi^{\prime \prime}\right)$ are two decompositions of the same pointed surface $(\Sigma, P, V)$, which are related by one of the changes $\mathcal{M}$ to $S$. Let $L^{\prime}$ and $L^{\prime \prime}$ be the Lagrangian subspaces generated by the $C^{\prime}$ and $C^{\prime \prime}$, respectively. In the cases $\mathcal{M}$ to $\mathcal{F}$, we have that $L^{\prime}=L^{\prime \prime}$, which is not the case for $S$. Let $\Sigma^{\prime}=\left(\Sigma, P, V, L^{\prime}\right)$ and $\Sigma^{\prime \prime}=\left(\Sigma, P, V, L^{\prime \prime}\right)$. We then get an induced isomorphism

$$
Z\left(\left(C^{\prime}, \Pi^{\prime}\right),\left(C^{\prime \prime}, \Pi^{\prime \prime}\right)\right): \bigoplus_{\mu \in \Lambda_{c^{\prime}}} Z(\lambda, \mu) \longrightarrow \bigoplus_{\mu \in \Lambda_{c^{\prime \prime}}} Z(\lambda, \mu)
$$

given by

$$
Z\left(\left(C^{\prime}, \Pi^{\prime}\right),\left(C^{\prime \prime}, \Pi^{\prime \prime}\right)\right)=Z\left(C^{\prime \prime}, \Pi^{\prime \prime}\right)^{-1} Z\left(\operatorname{Id}:\left(\Sigma^{\prime}, \lambda\right) \longrightarrow\left(\Sigma^{\prime \prime}, \lambda\right)\right) Z\left(C^{\prime}, \Pi^{\prime}\right) .
$$

This isomorphism is determined by the basic data via a finite sequence of the following changes.

$\mathcal{M}$ The linear map is given by a direct sum of tensor products of the linear maps induced by the morphism $\left(\Pi^{\prime \prime}\right)^{-1} \circ \Pi^{\prime}$ between the appropriate $\Xi$ 's, $\Delta$ 's and $\Upsilon$ 's.

$\mathcal{A}$ The linear map is induced by insertion or "removal" of the vector $\zeta(\lambda) \in Z_{\lambda, \lambda^{\dagger}}$ for the appropriate $\Xi$.

$\mathcal{D}$ The linear map is induced by tensoring the identity with the isomorphism (3) (or its inverse) inserted at the appropriate place.

$\mathcal{F}$ The linear map is induced by tensoring the identity with the isomorphism (1) inserted at the appropriate place. 
$\delta$ The linear map is induced by tensoring the identity with the isomorphism (2) inserted at the appropriate place.

Let $\left(C_{1}, \Pi_{1}\right)$ and $\left(C_{2}, \Pi_{2}\right)$ be any two decompositions of the same labeled pointed surface $(\Sigma, P, V, \lambda)$. Let $L_{i}$ be the induced Lagrangian subspaces on $\Sigma$ and $\Sigma_{i}=$ $\left(\Sigma, P, V, L_{i}\right)$ the corresponding marked surfaces. Since $\left(C_{1}, \Pi_{1}\right)$ and $\left(C_{2}, \Pi_{2}\right)$ are related by a sequence of changes $\mathcal{M}$ to $S$, we get that the isomorphism

$$
Z\left(\left(C_{1}, \Pi_{1}\right),\left(C_{2}, \Pi_{2}\right)\right): \bigoplus_{\mu_{1} \in \Lambda_{c_{1}}} Z\left(\lambda, \mu_{1}\right) \rightarrow \bigoplus_{\mu_{2} \in \Lambda_{c_{2}}} Z\left(\lambda, \mu_{2}\right)
$$

given by

$$
Z\left(\left(C_{1}, \Pi_{1}\right),\left(C_{2}, \Pi_{2}\right)\right)=Z\left(C_{2}, \Pi_{2}\right)^{-1} Z\left(\operatorname{Id}:\left(\Sigma_{1}, \lambda\right) \rightarrow\left(\Sigma_{2}, \lambda\right)\right) Z\left(C_{1}, \Pi_{1}\right)
$$

is also determined by the basic data.

Suppose now that $f:\left(\Sigma_{1}, \lambda_{1}\right) \rightarrow\left(\Sigma_{2}, \lambda_{2}\right)$ is a morphism of marked surfaces and $(C, \Pi)$ is a decomposition of $\boldsymbol{\Sigma}_{1}$. Then $(f(C), f \circ \Pi)$ is a decomposition of $\Sigma_{2}$, and we get a commutative diagram:

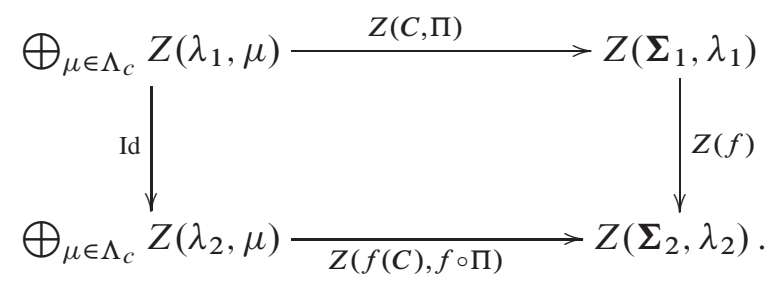

Suppose now $(\gamma, p)$ is an oriented, simple, closed curve with a preferred point $p$ on a marked surface $\boldsymbol{\Sigma}$. Let $\tilde{\boldsymbol{\Sigma}}$ be obtained from $\boldsymbol{\Sigma}$ by factoring along $(\gamma, p)$. Suppose that $(C, \Pi)$ is a decomposition of $\Sigma$, such that $\gamma \in C$. Then $(C, \Pi)$ also induces a decomposition of $\tilde{\boldsymbol{\Sigma}}$, say $(\widetilde{\mathrm{C}}, \widetilde{\Pi})$ and we get the following commutative diagram

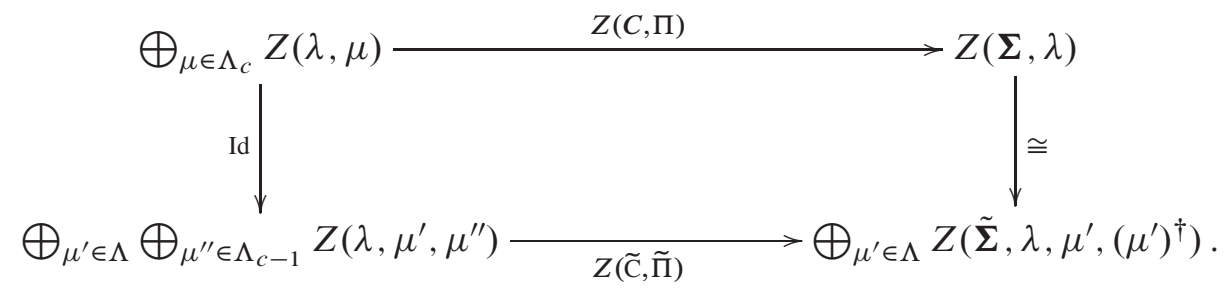

Likewise we trivially get a similar diagram for the case of the disjoint union isomorphism.

From this, it follows that the basic data determines the modular functor because the action of any morphism, the factorization and the disjoint union isomorphisms are all determined by the basic data.

By considering various finite sequences of decompositions of certain marked surfaces, we generate nontrivial relations on the basic data. Ten such universal relations are given on p. 55 in [34]. We will use some of them in Section 7. 


\section{Curve operators}

Let $\boldsymbol{\Sigma}=(\Sigma, P, V, L)$ be a general marked surface. Let $\lambda$ be a labeling of $\boldsymbol{\Sigma}$.

Let $\gamma$ be an oriented, simple, closed curve on $\Sigma-P$ and $\lambda_{\gamma} \in \Lambda$ a fixed label. We now define an operator

$$
Z\left(\gamma, \lambda_{\gamma}\right): Z(\Sigma, \lambda) \longrightarrow Z(\Sigma, \lambda)
$$

canonically associated to the pair $\left(\gamma, \lambda_{\gamma}\right)$.

Choose an embedding $\imath: D \rightarrow \Sigma-P$ of the unit disc $D$ into $\Sigma-P$, such that $\imath([-1,1])=\gamma \cap \imath(D)$ as indicated in Figure 4. Let $\left(\gamma_{l}, p_{l}\right)=(l(\partial D), l(1))$. Let $P^{\prime}=\left\{l\left(-\frac{1}{2}\right), l\left(\frac{1}{2}\right)\right\}$ and $V^{\prime}$ be directions along $\gamma$ in the positive direction at $P^{\prime}$. Let $\widetilde{P}=P \cup P^{\prime}, \widetilde{V}=V \cup V^{\prime}$ and $\tilde{\Sigma}=(\Sigma, \widetilde{P}, \widetilde{V}, L)$.

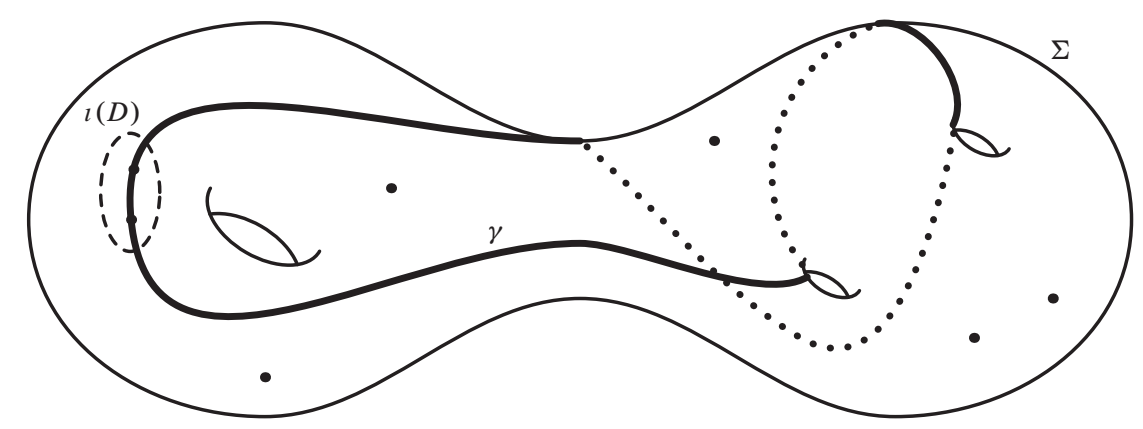

Figure 4. The curve $\gamma$ and the disk $l(D)$ on $\Sigma$.

The factorization of $\tilde{\Sigma}$ along $\gamma_{l}$ has two connected components which we denote $\widetilde{\Sigma}^{\prime}$ and $\widetilde{\Sigma}^{\prime \prime}$. Here, $\widetilde{\Sigma}^{\prime}$ is obtained from $l(D)$ by identifying $\gamma_{l}$ to a point $p^{\prime}$ with $p_{l} \in \gamma_{l}$ inducing a tangent direction $v^{\prime}$ at $p^{\prime}$. Likewise, $\widetilde{\Sigma}^{\prime \prime}$ is the quotient of $\Sigma-\imath(D-\partial D)$, where we identify $\gamma_{l}$ to a point $p^{\prime \prime}$, again with $p_{l} \in \gamma_{l}$ inducing a tangent direction $v^{\prime \prime}$ at $p^{\prime \prime}$.

Let $\widetilde{P}^{\prime}=P^{\prime} \cup\left\{p^{\prime}\right\}$ and $\widetilde{V}^{\prime}=V^{\prime} \cup\left\{v^{\prime}\right\}$, set $\tilde{\Sigma}^{\prime}=\left(\widetilde{\Sigma}^{\prime}, \widetilde{P}^{\prime}, \tilde{V}^{\prime}\right)$. Let $\widetilde{P}^{\prime \prime}=$ $P \cup\left\{p^{\prime \prime}\right\}$ and $\widetilde{V}^{\prime \prime}=V \cup\left\{v^{\prime \prime}\right\}$, set $\tilde{\boldsymbol{\Sigma}}^{\prime \prime}=\left(\widetilde{\Sigma}^{\prime \prime}, \widetilde{P}^{\prime \prime}, \widetilde{V}^{\prime \prime}\right)$.

The gluing and disjoint union axiom give isomorphisms

$$
Z\left(\tilde{\boldsymbol{\Sigma}}, \lambda, \lambda_{\gamma}^{\dagger}, \lambda_{\gamma}\right) \cong \bigoplus_{\mu \in \Lambda} Z\left(\tilde{\boldsymbol{\Sigma}}^{\prime}, \lambda_{\gamma}^{\dagger}, \lambda_{\gamma}, \mu\right) \otimes Z\left(\tilde{\boldsymbol{\Sigma}}^{\prime \prime}, \mu^{\dagger}, \lambda\right)
$$

and

$$
Z\left(\tilde{\Sigma}, \lambda, \lambda_{\gamma}, \lambda_{\gamma}^{\dagger}\right) \cong \bigoplus_{\mu \in \Lambda} Z\left(\tilde{\boldsymbol{\Sigma}}^{\prime}, \lambda_{\gamma}, \lambda_{\gamma}^{\dagger}, \mu\right) \otimes Z\left(\tilde{\boldsymbol{\Sigma}}^{\prime \prime}, \mu^{\dagger}, \lambda\right)
$$


The embedding $l$ induces an isomorphism of marked curves $l: \Upsilon \rightarrow \tilde{\boldsymbol{\Sigma}}^{\prime}$, which therefore gives isomorphisms

$$
Z(\imath): Z\left(\Upsilon, \lambda_{\gamma}^{\dagger}, \lambda_{\gamma}, 0\right) \longrightarrow Z\left(\tilde{\boldsymbol{\Sigma}}^{\prime}, \lambda_{\gamma}^{\dagger}, \lambda_{\gamma}, 0\right)
$$

and

$$
Z(l)^{\dagger}: Z\left(\tilde{\boldsymbol{\Sigma}}^{\prime}, \lambda_{\gamma}, \lambda_{\gamma}^{\dagger}, 0\right)^{*} \longrightarrow Z\left(\Upsilon, \lambda_{\gamma}, \lambda_{\gamma}^{\dagger}, 0\right)^{*}
$$

Also, by the gluing and disjoint union axiom combined with axiom MF4, we get an isomorphism

$$
Z\left(\tilde{\boldsymbol{\Sigma}}^{\prime \prime}, 0, \lambda\right) \cong Z(\Sigma, \lambda)
$$

which is unique up to scale.

The vector $\zeta_{1}\left(\lambda_{\gamma}^{\dagger}, \lambda_{\gamma}, 0\right) \in Z\left(\Upsilon, \lambda_{\gamma}^{\dagger}, \lambda_{\gamma}, 0\right)$ together with the isomorphisms $Z(l)$ gives us an inclusion

$$
I_{l}\left(\lambda_{\gamma}\right): Z(\Sigma, \lambda) \longrightarrow Z\left(\tilde{\Sigma}, \lambda, \lambda_{\gamma}^{\dagger}, \lambda_{\gamma}\right)
$$

A vector $\alpha_{\gamma} \in Z\left(\Upsilon, \lambda_{\gamma}, \lambda_{\gamma}^{\dagger}, 0\right)^{*}$ together with the isomorphism $Z(l)^{\dagger}$ gives a projection

$$
P_{l}\left(\lambda_{\gamma}\right): Z\left(\tilde{\boldsymbol{\Sigma}}, \lambda, \lambda_{\gamma}, \lambda_{\gamma}^{\dagger}\right) \longrightarrow Z(\Sigma, \lambda) .
$$

We shall normalize the forms $\alpha_{\mu} \in Z\left(\Upsilon, \mu, \mu^{\dagger}, 0\right)^{*}, \mu \in \Lambda$ as follows.

We require that ${ }^{1}$

$$
\alpha_{\mu}\left(Z(B)\left(\zeta_{1}(\mu \dagger, \mu, 0)\right)\right)=\frac{S_{0, \mu}}{S_{0,0}} .
$$

We shall now consider the following diffeomorphism $\Phi$ of $\tilde{\boldsymbol{\Sigma}}$. Fix a tubular neighborhood of $\gamma$ inside $\Sigma-P$. The diffeomorphism $\Phi$ will be the identity outside this tubular neighborhood. Inside the tubular neighborhood, it rotates and stretches $\imath\left(\left[-\frac{1}{2}, \frac{1}{2}\right]\right)$ onto $\gamma-\imath\left(\left(-\frac{1}{2}, \frac{1}{2}\right)\right)$ and $\gamma-\imath\left(\left(-\frac{1}{2}, \frac{1}{2}\right)\right)$ onto $l\left(\left[-\frac{1}{2}, \frac{1}{2}\right]\right)$. We see that

$$
Z(\Phi): Z\left(\tilde{\Sigma}, \lambda, \lambda_{\gamma}^{\dagger}, \lambda_{\gamma}\right) \longrightarrow Z\left(\tilde{\boldsymbol{\Sigma}}, \lambda, \lambda_{\gamma}, \lambda_{\gamma}^{\dagger}\right)
$$

Definition 4.1. The curve operator associated to $\left(\gamma, \lambda_{\gamma}\right)$ is by definition

$$
Z\left(\gamma, \lambda_{\gamma}\right)=P_{l}\left(\lambda_{\gamma}\right) \circ Z(\Phi) \circ I_{l}\left(\lambda_{\gamma}\right) .
$$

We observe that $Z\left(\gamma, \lambda_{\gamma}\right)$ does not depend on the choice of $\imath$. In fact, $Z\left(\gamma, \lambda_{\gamma}\right)$ only depends on the free homotopy class of $\gamma$.

We clearly have the following lemma.

Lemma 4.2. Suppose $\mathbf{f}: \boldsymbol{\Sigma}_{1} \rightarrow \boldsymbol{\Sigma}_{2}$ is a morphism of marked surfaces and $\gamma_{i}$ are closed, oriented curves on $\Sigma_{i}-P_{i}, i=1,2$, such that $f\left(\gamma_{1}\right)=\gamma_{2}$. Then

$$
Z(\mathbf{f})^{-1} Z\left(\gamma_{2}, \lambda\right) Z(\mathbf{f})=Z\left(\gamma_{1}, \lambda\right)
$$

for all $\lambda \in \Lambda$.

\footnotetext{
${ }^{1}$ We see that $S_{0,0} \neq 0$ by Corollary 7.1 .
} 
If $\gamma$ is contractible on the marked surfaces $\Sigma$, then

$$
Z\left(\gamma, \lambda_{\gamma}\right)=\frac{S_{0, \lambda_{\gamma}}}{S_{0,0}} \operatorname{Id}_{Z(\Sigma, \lambda)} .
$$

\section{The relation between curve operators and Dehn-twists}

In this section, we give a formula for the Dehn-twist operator in terms of the curve operators associated to any oriented, simple, closed curve.

Let $(\Sigma, \lambda)$ be a labeled marked surface. Let $\gamma$ be an oriented, simple, closed curve on $\Sigma-P$. Let $\varphi_{\gamma}:(\Sigma, \lambda) \rightarrow(\Sigma, \lambda)$ be the Dehn twist in the curve $\gamma$. By construction $\varphi_{\gamma}$ is the identity outside some tubular neighborhood of $\gamma$. Similarly, $Z\left(\gamma, \lambda_{\gamma}\right)$ is also a local construction within a tubular neighborhood of $\gamma$. Pick a point $p$ on $\gamma$ and let $\tilde{\Sigma}$ be obtained from $\Sigma$, by factoring $\Sigma$ along $(\gamma, p)$. Then we get an isomorphism

$$
Z(\Sigma, \lambda) \cong \bigoplus_{\mu} Z\left(\tilde{\Sigma}, \lambda, \mu, \mu^{\dagger}\right) .
$$

Both of the operators $Z\left(\varphi_{\gamma}\right)$ and $Z\left(\gamma, \lambda_{\gamma}\right)$ are diagonal with respect to this direct sum decomposition and act by multiples of the identity on each of the summands. This follows immediately from factoring along the boundary of a tubular neighborhood of $\gamma$. One also sees this way that these multiples by which these operators act by are independent of both $\gamma$ and $(\Sigma, \lambda)$.

Proposition 5.1. There exist uniquely determined constants $c_{\lambda \gamma} \in \mathbb{C}$ such that for any simple closed oriented curve $\gamma$ on any labeled marked surface $(\Sigma, \lambda)$ we have that

$$
Z\left(\varphi_{\gamma}\right)=\sum_{\lambda_{\gamma} \in \Lambda} c_{\lambda_{\gamma}} Z\left(\gamma, \lambda_{\gamma}\right) .
$$

Recall that $\Xi=\left(S^{2} ; 0, \infty ; v_{0}, v_{\infty}\right)$ and let $\gamma$ be the unit circle oriented in the positive direction.

We define $C_{\lambda, \mu} \in \mathbb{C}$ to be the scalar by which $Z(\gamma, \lambda)$ acts on $Z\left(\Xi, \mu, \mu^{\dagger}\right)=$ $Z_{\mu, \mu^{\dagger}}$, for $\lambda, \mu \in \Lambda$. Further, we recall that $d_{\mu} \in \mathbb{C}$ is the scalar by which $Z\left(\varphi_{\gamma}\right)$ acts on $Z\left(\Xi, \mu, \mu^{\dagger}\right)=Z_{\mu, \mu^{\dagger}}$.

Proposition 5.2. The matrix $C_{\lambda, \mu}$ is invertible.

We will prove this proposition in Section 7.

Proof of Proposition 5.1. The constants $c_{\lambda}$, which we seek has to satisfy

$$
d_{\mu}=\sum_{\lambda} c_{\lambda} C_{\lambda, \mu}
$$


By Proposition 5.2, the matrix $C$ is invertible, so there is a unique solution to this set of equations.

We remark that since $C$ is invertible, the $c_{\lambda}$ are determined by the genus zero data. Eq. (9) below gives an explicit formula for the $c_{\lambda}$ 's.

\section{The reduction from a once punctured genus one surfaces to genus zero}

Recall the simple, closed curves $\alpha$ and $\beta$ on $\Theta-\{p\}$ from Figure 3. Let $S: \Theta^{\alpha} \rightarrow \Theta^{\alpha}$ be the morphism of marked surfaces, which satisfies that

$$
S(\alpha)=\beta, \quad S(\beta)=\alpha^{-1},
$$

where we here interpret $\alpha$ and $\beta$ as generators of the fundamental group of $\Theta-\{p\}$ based at their intersection point.

Theorem 6.1. The morphism

$$
Z(S)=Z\left(\Theta^{\alpha}, \lambda\right) \longrightarrow Z\left(\Theta^{\alpha}, \lambda\right)
$$

is determined by the genus zero part of $Z$ for all $\lambda \in \Lambda$.

Proof. We recall that the mapping class group $\Gamma$ of $\Theta-\{p\}$ is

$$
\Gamma \cong\left\{S, T \mid(S T)^{3}=S^{2}\right\},
$$

where $S$ is as specified above and $T$ is the Dehn-twist in $\alpha$.

We see that

$$
S=T^{-1} S T^{-1} S^{-1} T^{-1}
$$

in the mapping class group $\Gamma$. It is easily checked that this gives the relation

$$
S=T^{-1} S T^{-1} S^{-1} T^{-1} \circ(\mathrm{Id},-1)
$$

in $\Gamma\left(\Theta^{\alpha}\right)$. Hence, if we let $T^{\prime}$ be the Dehn-twist in $\beta$, then

$$
S=T^{-1}\left(T^{\prime}\right)^{-1} T^{-1} \circ(\mathrm{Id},-1) .
$$

But by Proposition 5.1, we have that there are constants $\tilde{c}_{\lambda}$ such that

$$
Z\left(T^{\prime}\right)^{-1}=\sum_{\lambda_{\beta} \in \Lambda} \tilde{\mathrm{c}}_{\lambda_{\beta}} Z\left(\beta, \lambda_{\beta}\right) .
$$

So

$$
Z(S)=\varepsilon^{-1} \sum_{\lambda_{\beta} \in \Lambda} \tilde{\mathrm{c}}_{\lambda_{\beta}} Z\left(T^{-1}\right) Z\left(\beta, \lambda_{\beta}\right) Z\left(T^{-1}\right)
$$


Let $J: A \rightarrow \Theta-\{p\}$ be an embedding of an annulus $A$ into $\Theta-\{p\}$ as shown in Figure 5.

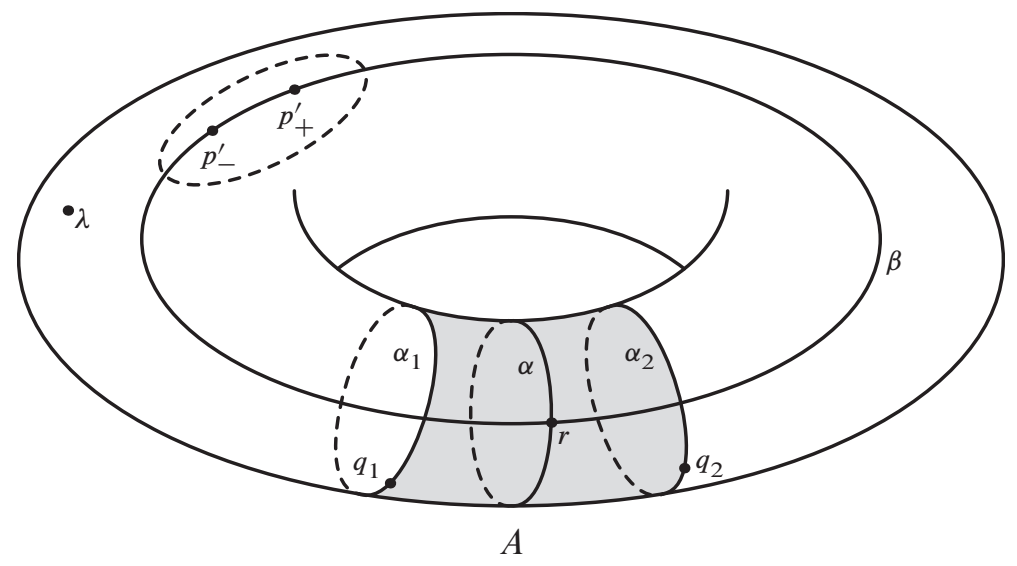

Figure 5. A once punctured surface of genus one with an annulus $A$ around the $\alpha$ curve.

Let $\alpha_{1}$ and $\alpha_{2}$ be the two boundary curves of $J(A)$, with base points say $q_{1} \in \alpha_{1}$ and $q_{2} \in \alpha_{2}$. Let $\Theta_{1}$ and $\Theta_{2}$, respectively, be obtained from $\Theta$ by factoring along $\alpha_{1}$ and $\alpha_{2}$, respectively. We denote the resulting base point and tangent direction by $\left(P_{1}, V_{1}\right)=\left(\left\{q_{1}^{\prime}, q_{1}^{\prime \prime}\right\},\left\{v_{q_{1}^{\prime}}, v_{q_{1}^{\prime \prime}}\right\}\right)$ and $\left(P_{2}, V_{2}\right)=\left(\left\{q_{2}^{\prime}, q_{2}^{\prime \prime}\right\},\left\{v_{q_{2}^{\prime}}, v_{q_{2}^{\prime \prime}}\right\}\right)$ on $\Theta_{1}$ and $\Theta_{2}$, respectively. Let $\widetilde{\Sigma}$ be obtained from $\Theta_{1}$, by factoring along $\alpha_{2}$ or equivalently from $\Theta_{2}$ by factoring along $\alpha_{1}$. Pick a point $r \in J(A-\partial A) \cap \gamma$ and the tangent direction $v_{r}$ at $r$ along $\beta$ in the positive direction.

Now choose an embedding $l: D \rightarrow \Theta-(J(A) \cup\{p\})$ and a diffeomorphism $\Phi$ with the properties required in the construction of $Z\left(\beta, \lambda_{\beta}\right)$. In fact we will choose a $\Phi$ which is a composite of two diffeomorphisms $\Phi_{1}$ and $\Phi_{2}$ as follows. Let $p_{-}^{\prime}=\imath\left(-\frac{1}{2}\right)$ and $p_{+}^{\prime}=\imath\left(\frac{1}{2}\right)$ and $v_{p_{-}^{\prime}}$ and $v_{p_{+}^{\prime}}$, respectively, be the induced tangent directions along $\beta$.

Let $P=\left\{p, p_{-}^{\prime}, p_{+}^{\prime}\right\}$ and $V=\left\{v_{p}, v_{p_{-}^{\prime}}, v_{p_{+}^{\prime}}\right\}$. Let $P^{\prime}=\left\{p, p_{+}^{\prime}, r\right\}$ and $V^{\prime}=\left\{v_{p}, v_{p_{+}^{\prime}}, v_{r}\right\}$. Further set

$$
\begin{array}{cc}
\widetilde{P}_{i}=P \cup P_{i}, & \widetilde{V}_{i}=V \cup V_{i}, \\
\widetilde{P}_{i}^{\prime}=P^{\prime} \cup P_{i}, & \widetilde{V}_{i}^{\prime}=V^{\prime} \cup V_{i},
\end{array}
$$

for $i=1,2$.

We pick

$$
\Phi_{1}:\left(\Theta_{2}, \widetilde{P}_{2}, \widetilde{V}_{2}\right) \longrightarrow\left(\Theta_{2}, \widetilde{P}_{2}^{\prime}, \tilde{V}_{2}^{\prime}\right)
$$

such that $\Phi_{1}$ is the identity outside a tubular neighborhood of the piece of $\beta$ from $p_{-}^{\prime}$ 
to $r$ and it maps $\Phi_{1}\left(p_{-}^{\prime}\right)=p_{+}^{\prime}$ and $\Phi_{1}\left(p_{+}^{\prime}\right)=r$. The map

$$
\Phi_{2}:\left(\Theta_{1}, \widetilde{P}_{1}^{\prime}, \tilde{V}_{1}^{\prime}\right) \longrightarrow\left(\Theta_{1}, \widetilde{P}_{1}, \tilde{V}_{1}\right)
$$

is chosen such that it is the identity outside a neighborhood of the piece of $\beta$ from $r$ to $p_{-}^{\prime}$ and it maps $\Phi_{2}(r)=p_{-}^{\prime}$. By re-gluing $\Theta_{1}$ and $\Theta_{2}$ to obtain $\Theta$, we see that $\Phi_{1}$ and $\Phi_{2}$ induce diffeomorphisms $\Phi_{1}^{\prime}$ and $\Phi_{2}^{\prime}$ of $\Theta$, which preserve $\alpha_{2}$ and $\alpha_{1}$, respectively. We let

$$
\Phi=\Phi_{2}^{\prime} \circ \Phi_{1}^{\prime}:(\Theta, P, V) \longrightarrow(\Theta, P, V) .
$$

Thus we have the linear maps for all choices of $\mu, \mu^{\prime} \in \Lambda$,

$$
Z\left(\Phi_{1}\right)_{\mu}: Z\left(\Theta_{2}, \widetilde{P}_{2}, \tilde{V}_{2}, \lambda, \lambda_{\gamma}^{\dagger}, \lambda_{\gamma}, \mu, \mu^{\dagger}\right) \longrightarrow Z\left(\Theta_{2}, \widetilde{P}_{2}^{\prime}, \tilde{V}_{2}^{\prime}, \lambda, \lambda_{\gamma}^{\dagger}, \lambda_{\gamma}, \mu, \mu^{\dagger}\right)
$$

and

$$
\begin{aligned}
Z\left(\Phi_{2}\right)_{\mu^{\prime}}: Z\left(\Theta_{1}, \widetilde{P}_{1}^{\prime}, \widetilde{V}_{1}^{\prime}, \lambda, \lambda_{\gamma}^{\dagger}, \lambda \gamma, \mu^{\prime},\left(\mu^{\prime}\right)^{\dagger}\right) & \\
& \longrightarrow Z\left(\Theta_{1}, \widetilde{P}_{1}, \widetilde{V}_{1}, \lambda, \lambda_{\gamma}, \lambda_{\gamma}^{\dagger}, \mu^{\prime},\left(\mu^{\prime}\right)^{\dagger}\right) .
\end{aligned}
$$

We further have the following two commutative diagrams:

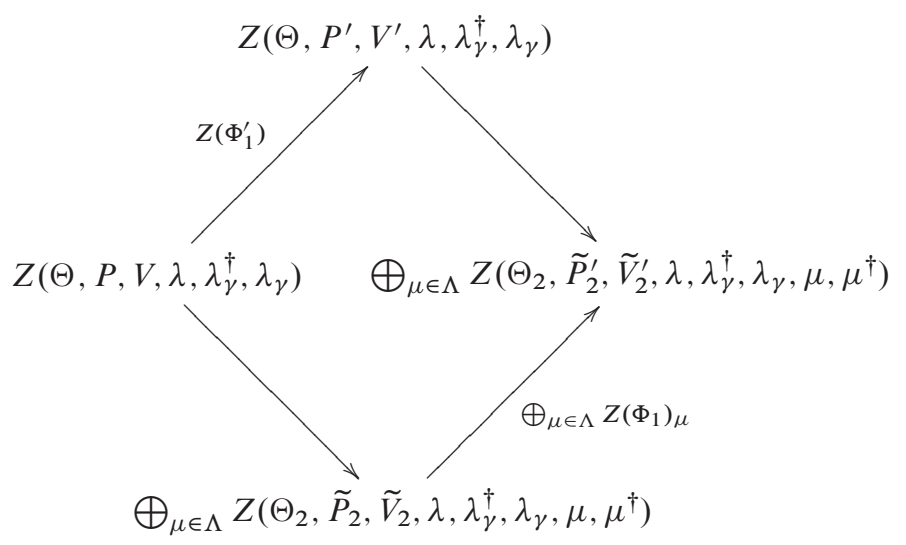

and

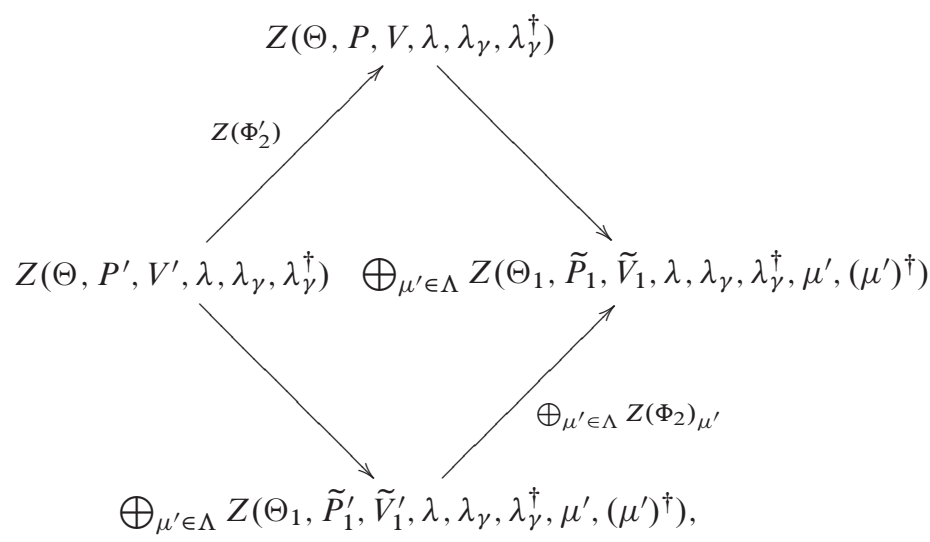


where the vertical arrows are the factorization isomorphisms.

By the commutativity of factorization, we also have the following commutative diagram of isomorphisms:

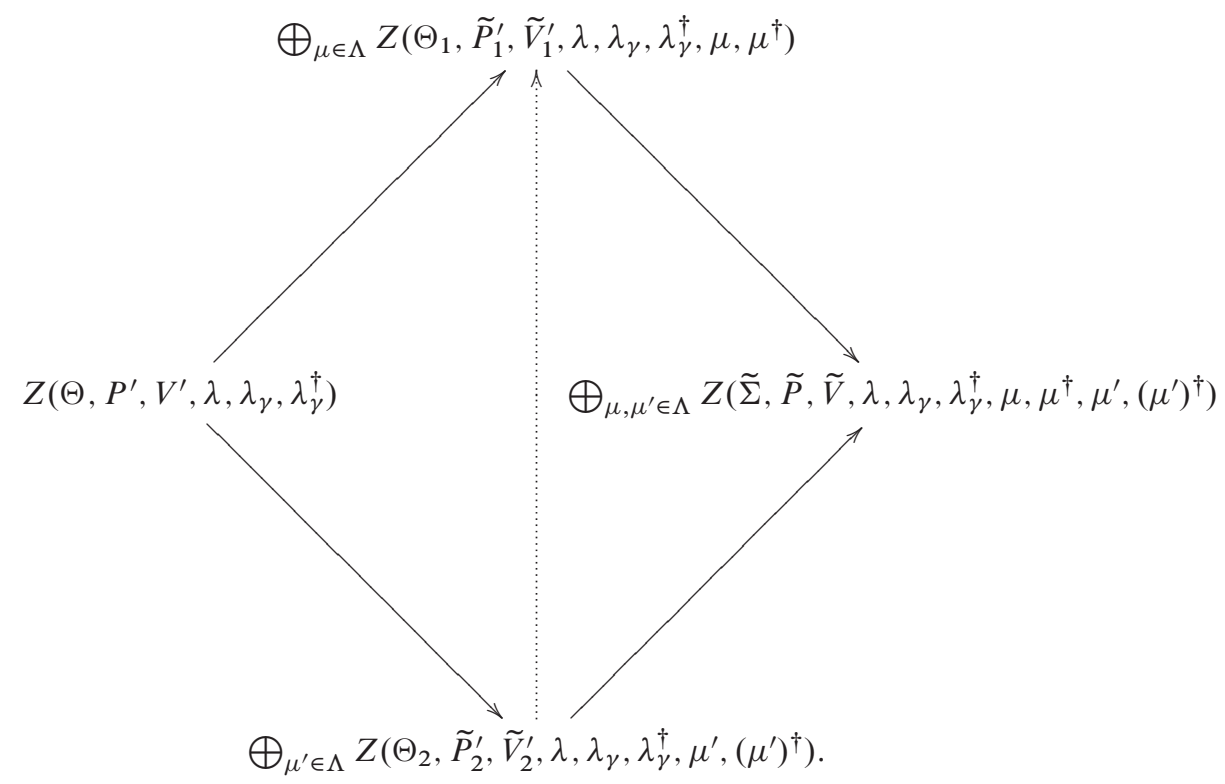

Now consider the curve operator $Z\left(\beta, \lambda_{\beta}\right)$. Since factorization along non-intersecting curves commute, we see that both $P_{l}\left(\lambda_{\beta}\right)$ and $I_{l}\left(\lambda_{\beta}\right)$ are determined by genus zero morphism by factoring along say $\alpha_{1}$, which commutes with the factorization along $\gamma_{l}$.

By representing $T$ as the Dehn-twist in $\alpha_{1}$ and $\alpha_{2}$, respectively,we get diffeomorphisms

$$
T_{1}:\left(\Theta_{2}, \widetilde{P}_{2}^{\prime}, \tilde{V}_{2}^{\prime}\right) \longrightarrow\left(\Theta_{2}, \widetilde{P}_{2}^{\prime}, \tilde{V}_{2}^{\prime}\right)
$$

and

$$
T_{2}:\left(\Theta_{1}, \widetilde{P}_{1}^{\prime}, \widetilde{V}_{1}^{\prime}\right) \longrightarrow\left(\Theta_{1}, \widetilde{P}_{1}^{\prime}, \widetilde{V}_{1}^{\prime}\right) .
$$

Since both of these diffeomorphisms are the identity in a neighborhood of $\iota(D)$, we get the equation

$$
Z(S)=\varepsilon^{-1} \sum_{\lambda_{\beta} \in \Lambda} \tilde{\mathrm{c}}_{\lambda_{\beta}} P_{l}\left(\lambda_{\beta}\right) Z\left(T_{2}^{-1} \Phi_{2}^{\prime}\right) Z\left(\Phi_{1}^{\prime} T_{1}^{-1}\right) I_{l}\left(\lambda_{\beta}\right) .
$$

Tracing through the previous three commutative diagrams, we see that $Z\left(\Phi_{1}^{\prime} T_{1}^{-1}\right)$ and $Z\left(T_{2}^{-1} \Phi_{2}^{\prime}\right)$ are determined by genus zero data. 
We observe that the same argument can be used to show that the action of a Dehntwist along any curve on a marked surface equipped with a fixed decomposition is determined by the isomorphisms $\mathcal{M}$ to $\mathscr{F}$.

Proof of Theorem 1.1. We consider the morphism

$$
S:\left(\Theta^{\alpha}, \lambda\right) \longrightarrow\left(\Theta^{\beta}, \lambda\right)
$$

and observe that it is compatible with the factorizations in $\alpha$ and $\beta$ and via $f_{\alpha}$ and $f_{\beta}$ is compatible with the identity morphism Id : $\Upsilon \rightarrow \Upsilon$. We have the composition identity

$$
S:\left(\Theta^{\alpha}, \lambda\right) \longrightarrow\left(\Theta^{\beta}, \lambda\right)\left(\operatorname{Id}:\left(\Theta^{\alpha}, \lambda\right) \longrightarrow\left(\Theta^{\beta}, \lambda\right)\right) \circ\left(S:\left(\Theta^{\alpha}, \lambda\right) \longrightarrow\left(\Theta^{\alpha}, \lambda\right)\right) .
$$

Here, we have taken the liberty to use the same letter $S$ to denote the two different morphisms, which, however, involve the same underlying diffeomorphism. From this, we conclude that

$$
Z\left(f_{\alpha}^{-1}\right) Z\left(S:\left(\Theta^{\alpha}, \lambda\right) \longrightarrow\left(\Theta^{\alpha}, \lambda\right)\right) Z\left(f_{\alpha}\right)=S(\lambda)^{-1} .
$$

Since $Z(S): Z\left(\Theta^{\alpha}, \lambda\right) \rightarrow Z\left(\Theta^{\alpha}, \lambda\right)$ is determined by genus zero data, we see that so is $S(\lambda)$ for all $\lambda \in \Lambda$.

\section{Consequences of the universal relations on the basic data}

In this section we will prove Proposition 5.2. It follows immediately from Proposition 7.6 below. However, we first need to derive a couple of consequences from the pentagon relation for $F$ (Figure 7) and the relations between $F$ and $S$ (Figure 6).

Consider the isomorphism (1). If one of the labels $\lambda, \mu, \xi, \kappa$ equals $0 \in \Lambda$, this is a special case. In this case, all of the terms in the direct sum of the domain and codomain of (1) are zero, except for one of them.

Lemma 7.1. We have the formulae:

$$
\begin{array}{ll}
\lambda=0, & F\left(\zeta\left(\mu^{\dagger}, 0, \mu\right) \otimes v=R^{2}(v) \otimes \zeta\left(\xi^{\dagger}, \xi, 0\right),\right. \\
\mu=0, & F\left(\zeta\left(\lambda^{\dagger}, \lambda, 0\right) \otimes v\right)=\zeta\left(\kappa^{\dagger}, 0, \kappa\right) \otimes R(v), \\
\xi=0, & F\left(v \otimes \zeta\left(\kappa^{\dagger}, \kappa, 0\right)\right)=R(v) \otimes \zeta\left(\lambda^{\dagger}, 0, \lambda\right), \\
\kappa=0, & F\left(v \otimes \zeta\left(\xi^{\dagger}, 0, \xi\right)\right)=\zeta\left(\mu^{\dagger}, \mu, 0\right) \otimes R^{2}(v) .
\end{array}
$$

Proof. The case $\xi=0$ is precisely Relation 5 on p. 55 of [34]. They are all obtained by considering the two ways of decomposing $\Upsilon$ along a system of two curves, related by an $\mathscr{F}$ change and such that the factorization of both gives two copies of $\Upsilon$ and one copy of $\Delta$, as illustrated in Figure 27 on p. 53 of [34]. 
For each $\lambda \in \Lambda$ we define

$$
E_{\lambda}=F_{0,0}\left[\begin{array}{cc}
\lambda & \lambda^{\dagger} \\
\lambda^{\dagger} & \lambda
\end{array}\right]
$$

Lemma 7.2. We have the following formula:

$$
S_{0,0} E_{\lambda}=S_{0, \lambda^{\dagger}}
$$

for all $\lambda \in \Lambda$.

Proof. We consider Relation 3 on p. 55 of [34], which is obtained from considering six decompositions of a genus one surface with two marked points as depicted in Figure 6. See also Figure 18 on p. 43 and Figure 26 on p. 52 of [34].

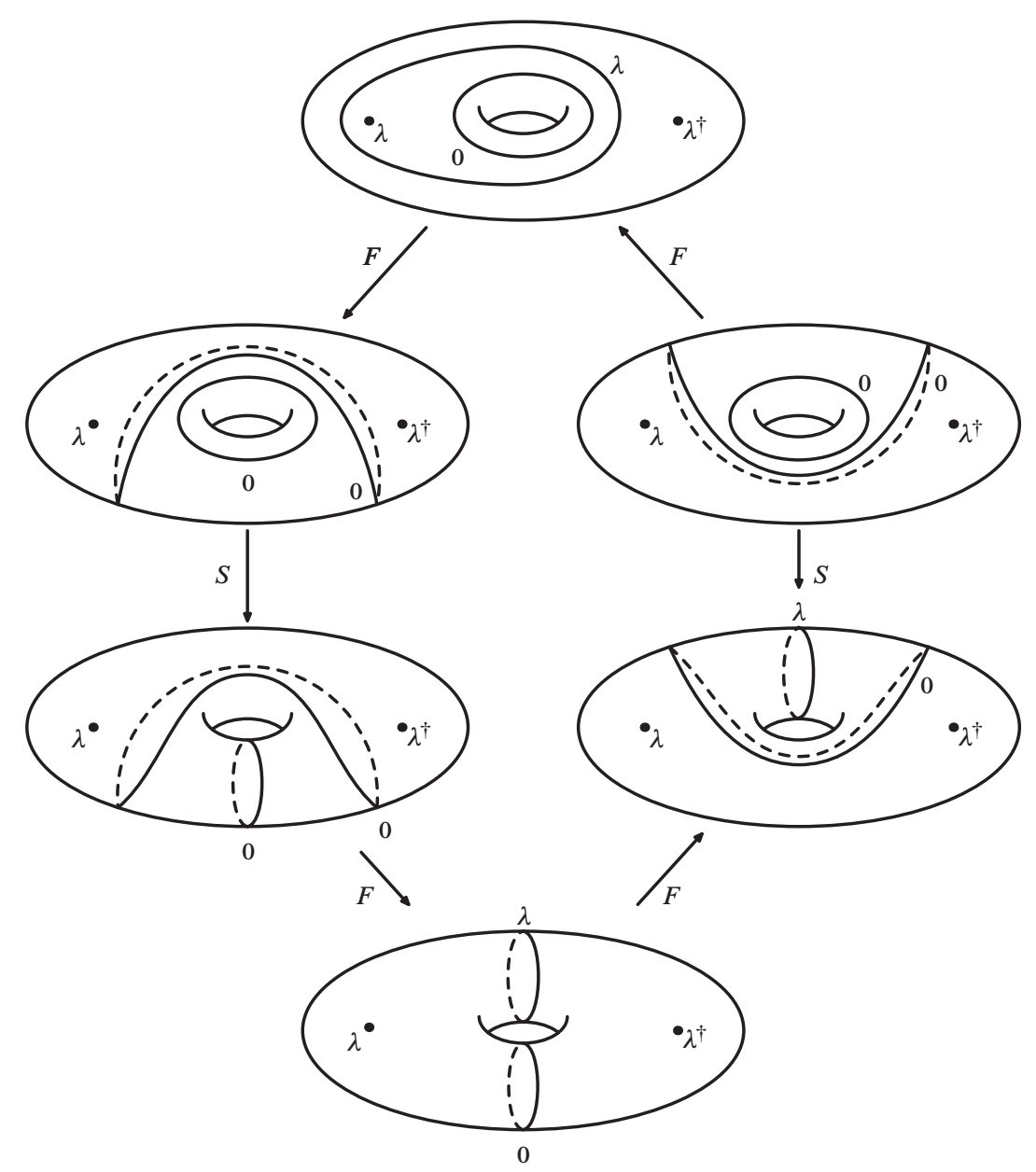

Figure 6. A relation between $S$ and $F$. 
Starting at the domain of the morphism $S$ on the right of Figure 6 and going counter clockwise around to the codomain of the same morphism, we get that

$$
\begin{aligned}
& \zeta\left(0, \lambda^{\dagger}, \lambda\right) \otimes \zeta(0,0,0) \\
& \longmapsto \zeta\left(\lambda^{\dagger}, \lambda, 0\right) \otimes \zeta\left(\lambda, 0, \lambda^{\dagger}\right) \\
& \stackrel{B_{23}^{-1} \otimes B_{23}^{-1}}{\longrightarrow} \zeta\left(\lambda^{\dagger}, 0, \lambda\right) \otimes \zeta\left(\lambda, \lambda^{\dagger}, 0\right) \\
& \longmapsto \zeta\left(0, \lambda, \lambda^{\dagger}\right) \otimes \zeta(0,0,0) \\
& \stackrel{\mathrm{Id} \otimes T_{3}^{-1} T_{1} B_{23}}{\longrightarrow} \zeta\left(0, \lambda, \lambda^{\dagger}\right) \otimes \zeta(0,0,0) \\
& \longmapsto S_{0,0} \zeta\left(0, \lambda, \lambda^{\dagger}\right) \otimes \zeta(0,0,0) \\
& \longmapsto S_{0,0} \zeta\left(\lambda, \lambda^{\dagger}, 0\right) \otimes \zeta\left(\lambda^{\dagger}, 0, \lambda\right) \\
& \stackrel{R^{-1} \otimes R}{\longrightarrow} S_{0,0} \zeta\left(0, \lambda, \lambda^{\dagger}\right) \otimes \zeta\left(0, \lambda, \lambda^{\dagger}\right) \\
& \longmapsto S_{0,0} E_{\lambda} \zeta\left(0, \lambda^{\dagger}, \lambda\right) \otimes \zeta\left(0, \lambda^{\dagger}, \lambda\right)
\end{aligned}
$$

Whereas the morphism $S$ of course gives

$$
\zeta\left(0, \lambda^{\dagger}, \lambda\right) \otimes \zeta(0,0,0) \stackrel{\operatorname{Id} \otimes S}{\longrightarrow} S_{0, \lambda \dagger} \zeta\left(0, \lambda^{\dagger}, \lambda\right) \otimes \zeta\left(0, \lambda^{\dagger}, \lambda\right)
$$

From this, we conclude the formula

$$
S_{0,0} E_{\lambda}=S_{0, \lambda^{\dagger}}
$$

Corollary 7.3. We have that $S_{0,0} \neq 0$ for any modular functor.

Lemma 7.4. For all $\lambda, \mu, v \in \Lambda$, we have the relation

$$
E_{\lambda^{\dagger}} \sum_{i, j, r} F_{0, \mu}\left[\begin{array}{cc}
\lambda & v \\
\lambda^{\dagger} & v^{\dagger}
\end{array}\right]_{11}^{i j} R_{j r} F_{v, 0}\left[\begin{array}{cc}
\lambda & \lambda^{\dagger} \\
\mu^{\dagger} & \mu
\end{array}\right]_{r l}^{11} R_{i s}^{2}=\delta_{s l}
$$

Proof. The pentagon relation, as depicted in Figure 7, gives a relation between five applications of the $F$-isomorphism between five decompositions of a genus zero surface with five marked points. This is Relation 1 on p. 55 of [34] which is also illustrated in Figure 24 on p. 50 of [34]. 


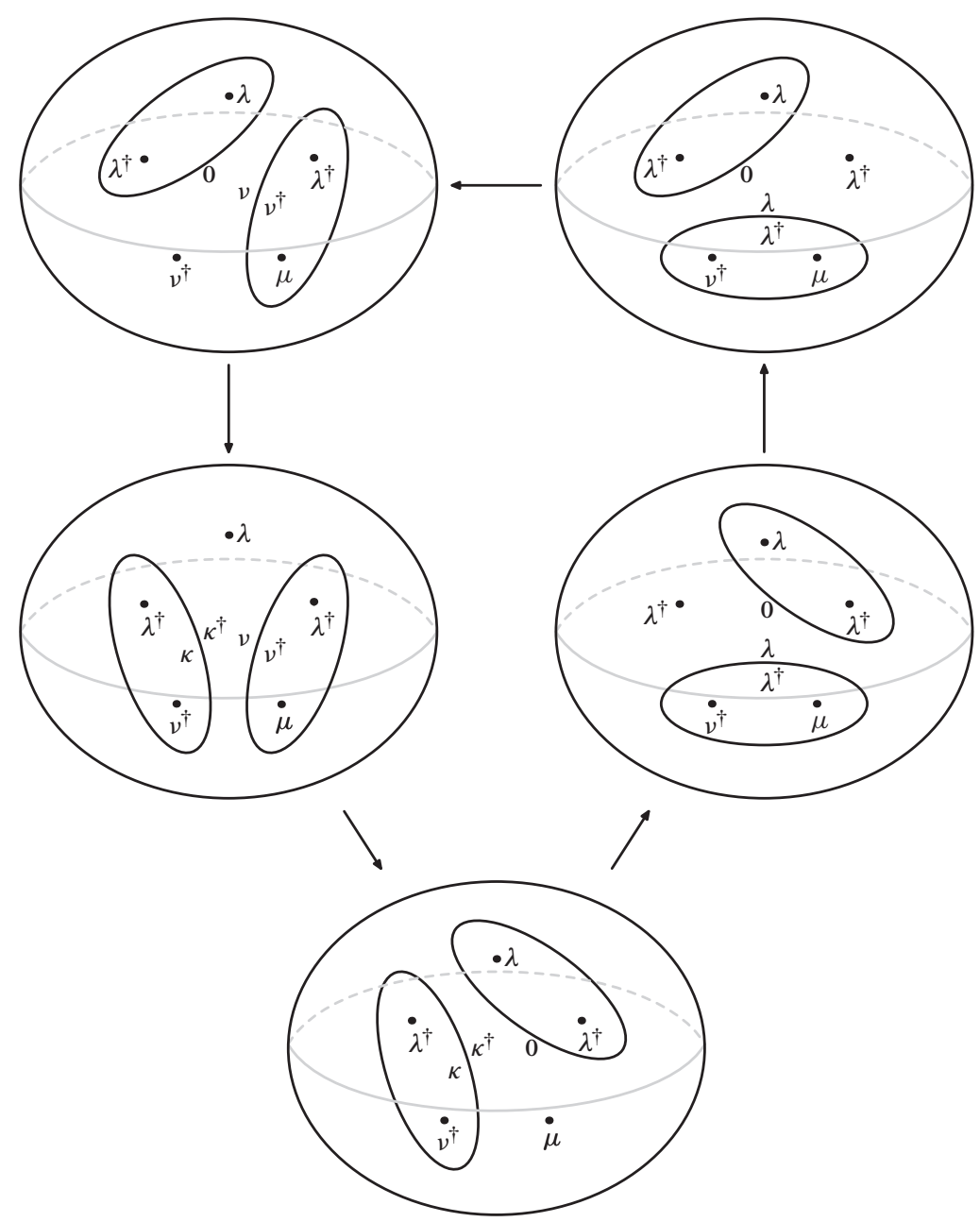

Figure 7. The pentagon relation for $F$.

Starting in the upper left-hand corner and going counter clockwise around, we get that

$$
\begin{aligned}
& \zeta\left(0, \lambda, \lambda^{\dagger}\right) \otimes \zeta\left(0, v, \nu^{\dagger}\right) \otimes \zeta_{l}\left(v^{\dagger}, \mu, \lambda^{\dagger}\right) \\
& \stackrel{F \otimes \mathrm{Id}}{\longmapsto} \sum_{\kappa, i, j} F_{0, \kappa}\left[\begin{array}{cc}
\lambda & v \\
\lambda^{\dagger} & v^{\dagger}
\end{array}\right]_{11}^{i j} \\
& \zeta_{i}\left(\kappa, \lambda^{\dagger}, \nu^{\dagger}\right) \otimes \zeta_{j}\left(\kappa^{\dagger}, v, \lambda\right) \otimes \zeta_{l}\left(v^{\dagger}, \mu, \lambda^{\dagger}\right)
\end{aligned}
$$




$$
\begin{aligned}
& \stackrel{\operatorname{Id} \otimes R \otimes \mathrm{Id}}{\longrightarrow} \sum_{\kappa, i, j, r} F_{0, \kappa}\left[\begin{array}{cc}
\lambda & v \\
\lambda^{\dagger} & v^{\dagger}
\end{array}\right]_{11}^{i j} R_{j r} \\
& \zeta_{i}\left(\kappa, \lambda^{\dagger}, v^{\dagger}\right) \otimes \zeta_{r}\left(v, \lambda, \kappa^{\dagger}\right) \otimes \zeta_{l}\left(v^{\dagger}, \mu, \lambda^{\dagger}\right) \\
& \stackrel{\mathrm{Id} \otimes F}{\longrightarrow} \sum_{\kappa, i, j, r} F_{0, \kappa}\left[\begin{array}{cc}
\lambda & v \\
\lambda^{\dagger} & v^{\dagger}
\end{array}\right]_{11}^{i j} R_{j r} F_{v, 0}\left[\begin{array}{cc}
\lambda & \lambda^{\dagger} \\
\kappa^{\dagger} & \mu
\end{array}\right]_{r l}^{11} \\
& \zeta_{i}\left(\kappa, \lambda^{\dagger}, v^{\dagger}\right) \otimes \zeta\left(\kappa^{\dagger}, \mu, 0\right) \otimes \zeta\left(0, \lambda^{\dagger}, \lambda\right) \\
& \stackrel{\mathrm{Id} \otimes R \otimes \mathrm{Id}}{\longrightarrow} \sum_{i, j, r} F_{0, \kappa}\left[\begin{array}{cc}
\lambda & v \\
\lambda^{\dagger} & v^{\dagger}
\end{array}\right]_{11}^{i j} R_{j r} F_{v, 0}\left[\begin{array}{cc}
\lambda & \lambda^{\dagger} \\
\kappa^{\dagger} & \mu
\end{array}\right]_{r l}^{11} \\
& \zeta_{i}\left(\mu, \lambda^{\dagger}, \nu^{\dagger}\right) \otimes \zeta\left(\mu^{\dagger}, \mu, 0\right) \otimes \zeta\left(0, \lambda^{\dagger}, \lambda\right) \\
& \stackrel{F \otimes \mathrm{Id}}{\longrightarrow} \sum_{i, j, r, s} F_{0, \kappa}\left[\begin{array}{cc}
\lambda & v \\
\lambda^{\dagger} & v^{\dagger}
\end{array}\right]_{11}^{i j} R_{j r} F_{v, 0}\left[\begin{array}{cc}
\lambda & \lambda^{\dagger} \\
\kappa^{\dagger} & \mu
\end{array}\right]_{r l}^{11} R_{i s} \\
& \zeta_{s}\left(\lambda^{\dagger}, v^{\dagger}, \mu\right) \otimes \zeta\left(\lambda, 0, \lambda^{\dagger}\right) \otimes \zeta\left(0, \lambda^{\dagger}, \lambda\right) \\
& \stackrel{\operatorname{Id} \otimes R \otimes I \mathrm{I}}{\longrightarrow} \sum_{i, j, r, s} F_{0, \kappa}\left[\begin{array}{cc}
\lambda & v \\
\lambda^{\dagger} & v^{\dagger}
\end{array}\right]_{11}^{i j} R_{j r} F_{v, 0}\left[\begin{array}{cc}
\lambda & \lambda^{\dagger} \\
\kappa^{\dagger} & \mu
\end{array}\right]_{r l}^{11} R_{i s} \\
& \zeta\left(0, \lambda^{\dagger}, \lambda\right) \otimes \zeta\left(0, \lambda^{\dagger}, \lambda\right) \otimes \zeta_{s}\left(\lambda^{\dagger}, v^{\dagger}, \mu\right) \\
& \stackrel{F \otimes \mathrm{Id}}{\longrightarrow} E_{\lambda^{\dagger}} \sum_{i, j, r, s} F_{0, \kappa}\left[\begin{array}{cc}
\lambda & v \\
\lambda^{\dagger} & v^{\dagger}
\end{array}\right]_{11}^{i j} R_{j r} F_{v, 0}\left[\begin{array}{cc}
\lambda & \lambda^{\dagger} \\
\kappa^{\dagger} & \mu
\end{array}\right]_{r l}^{11} R_{i s} \\
& \zeta\left(0, \lambda, \lambda^{\dagger}\right) \otimes \zeta\left(0, \lambda, \lambda^{\dagger}\right) \otimes \zeta_{s}\left(\lambda^{\dagger}, \nu^{\dagger}, \mu\right) \\
& \stackrel{\mathrm{Id} \otimes R \otimes \mathrm{Id}}{\longrightarrow} E_{\lambda^{\dagger}} \sum_{i, j, r, s} F_{0, \kappa}\left[\begin{array}{cc}
\lambda & v \\
\lambda^{\dagger} & v^{\dagger}
\end{array}\right]_{11}^{i j} R_{j r} F_{v, 0}\left[\begin{array}{cc}
\lambda & \lambda^{\dagger} \\
\kappa^{\dagger} & \mu
\end{array}\right]_{r l}^{11} R_{i s} \\
& \zeta\left(0, \lambda, \lambda^{\dagger}\right) \otimes \zeta\left(\lambda, \lambda^{\dagger}, 0\right) \otimes \zeta_{s}\left(\lambda^{\dagger}, v^{\dagger}, \mu\right) \\
& \stackrel{\mathrm{Id} \otimes F}{\longrightarrow} E_{\lambda^{\dagger}} \sum_{i, j, r, s} F_{0, \kappa} \lambda \nu \lambda^{\dagger} \nu^{\dagger_{11}^{i j}} R_{j r} F_{\nu, 0} \lambda \lambda^{\dagger} \kappa^{\dagger} \mu_{r l}^{11} R_{i s}^{2} \\
& \zeta\left(0, \lambda, \lambda^{\dagger}\right) \otimes \zeta\left(v, 0, v^{\dagger}\right) \otimes \zeta_{s}\left(v^{\dagger}, \mu, \lambda^{\dagger}\right) \\
& \stackrel{\mathrm{Id} \otimes R \otimes \mathrm{Id}}{\longrightarrow} E_{\lambda^{\dagger}} \sum_{i, j, r, s} F_{0, \kappa}\left[\begin{array}{cc}
\lambda & v \\
\lambda^{\dagger} & v^{\dagger}
\end{array}\right]_{11}^{i j} R_{j r} F_{v, 0}\left[\begin{array}{cc}
\lambda & \lambda^{\dagger} \\
\kappa^{\dagger} & \mu
\end{array}\right]_{r l}^{11} R_{i s}^{2} \\
& \zeta\left(0, \lambda, \lambda^{\dagger}\right) \otimes \zeta\left(0, v^{\dagger}, v\right) \otimes \zeta_{s}\left(v^{\dagger}, \mu, \lambda^{\dagger}\right) .
\end{aligned}
$$

This proves the stated equation.

From (5), we conclude that $E_{\lambda} \neq 0$ and, therefore, also that $S_{0, \lambda} \neq 0$ for all $\lambda \in \Lambda$. Consider now (5) as a product of two matrices, namely on the one hand the terms involving $E_{\lambda^{\dagger}}$, the first $F$ and $R^{2}$ and on the other the second $F$ and $R$. The 
equation states that these two matrices are each other's inverses. But then they also satisfy the equation where we multiply them in the other order:

$$
\sum_{l, u, v} F_{0, \mu}\left[\begin{array}{cc}
\lambda & v^{\dagger} \\
\lambda^{\dagger} & v
\end{array}\right]_{11}^{l m} R_{l u}^{2} F_{v^{\dagger}, 0}\left[\begin{array}{cc}
\lambda & \lambda^{\dagger} \\
\mu^{\dagger} & \mu
\end{array}\right]_{v u}^{11} R_{t v}=E_{\lambda^{\dagger}}^{-1} \delta_{t m} .
$$

By summing over $m$ in (6) we get

$$
\sum_{m, u, l, v} F_{0, \mu}\left[\begin{array}{cc}
\lambda & v^{\dagger} \\
\lambda^{\dagger} & v
\end{array}\right]_{11}^{l m} R_{l u}^{2} F_{v, 0}\left[\begin{array}{cc}
\lambda & \lambda^{\dagger} \\
\mu^{\dagger} & \mu
\end{array}\right]_{v u}^{11} R_{m v}=E_{\lambda^{\dagger}}^{-1} N_{v, \mu}^{\lambda}
$$

As above, $\Theta$ is an oriented genus one surface and $\alpha$ and $\beta$ are simple, closed curves, as indicated in Figure 3. Let $\boldsymbol{\Theta}_{\alpha}^{\prime}$ and $\boldsymbol{\Theta}_{\beta}^{\prime}$ be marked surfaces, which result from factoring $\left(\Sigma, L_{\alpha}\right)$ along $\alpha$ and $\left(\Sigma, L_{\beta}\right)$ along $\beta$, respectively. By factorization, we get isomorphisms

$$
\Phi_{\alpha}^{\prime}: Z\left(\Theta, L_{\alpha}\right) \longrightarrow \bigoplus_{\lambda} Z\left(\Theta_{\alpha}^{\prime}, \lambda, \lambda^{\dagger}\right)
$$

and

$$
\Phi_{\beta}^{\prime}: Z\left(\Theta, L_{\beta}\right) \longrightarrow \bigoplus_{\mu} Z\left(\boldsymbol{\Theta}_{\beta}^{\prime}, \mu, \mu^{\dagger}\right) .
$$

Pick diffeomorphisms

$$
f_{\alpha}^{\prime}: \Xi \longrightarrow \boldsymbol{\Theta}_{\alpha}^{\prime}
$$

and

$$
f_{\beta}^{\prime}: \Xi \longrightarrow \boldsymbol{\Theta}_{\beta}^{\prime}
$$

which map the real axis onto $\beta$ and $\alpha$, respectively.

Then we get a basis $\xi_{\lambda}^{\alpha}$ for $Z\left(\Sigma, L_{\alpha}\right)$ and $\xi_{\mu}^{\beta}$ for $Z\left(\Sigma, L_{\beta}\right)$ by

$$
\xi_{\lambda}^{\alpha}=\left(\Phi^{\prime}\right)_{\alpha}^{-1} Z\left(f_{\alpha}^{\prime}\right)\left(\xi_{\lambda}\right)
$$

and

$$
\xi_{\mu}^{\beta}=\left(\Phi^{\prime}\right)_{\beta}^{-1} Z\left(f_{\beta}^{\prime}\right)\left(\xi_{\mu}\right)
$$

We have of course that

$$
\zeta_{\lambda}^{\beta}=\sum_{\mu} S_{\lambda, \mu} \zeta_{\mu}^{\alpha}
$$

Proposition 7.5. We have the following equation:

$$
Z(\beta, \lambda) \xi_{\mu}^{\alpha}=\sum_{v} N_{\mu, \nu}^{\lambda} \xi_{v}^{\alpha}
$$


Proof. We compute the action of $Z(\beta, \lambda)$ on $\zeta_{\mu}^{\alpha}$ by computing the compositions indicated in Figure 8.

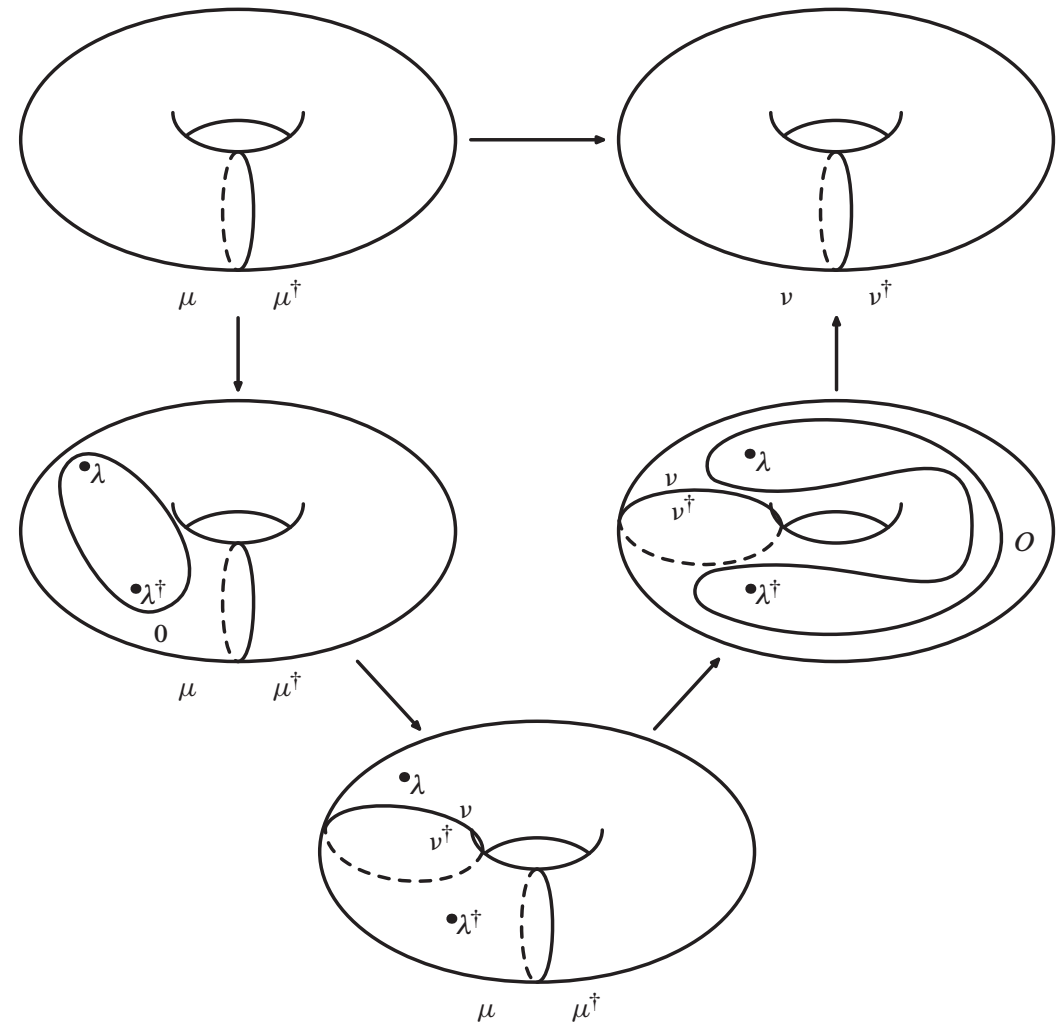

Figure 8 . The curve operator along $\beta$ on the genus one surface $\Theta$.

$$
\begin{aligned}
& \zeta_{\mu}^{\alpha} \longmapsto \zeta\left(0, \lambda, \lambda^{\dagger}\right) \otimes \zeta\left(0, \mu, \mu^{\dagger}\right) \\
& \longmapsto \sum_{\nu, i, j} F_{0, v}\left[\begin{array}{cc}
\lambda & \mu^{\dagger} \\
\lambda^{\dagger} & \mu
\end{array}\right]_{11}^{j i} \zeta_{j}\left(v, \lambda^{\dagger}, \mu\right) \otimes \zeta_{i}\left(v^{\dagger}, \mu^{\dagger}, \lambda\right) \\
& \stackrel{R^{2} \otimes R}{\longrightarrow} \sum_{\nu, i, j, r, t} F_{0, \nu}\left[\begin{array}{cc}
\lambda & \mu^{\dagger} \\
\lambda^{\dagger} & \mu
\end{array}\right]_{11}^{j i} R_{j r}^{2} R_{i t} \zeta_{r}\left(\mu, \nu, \lambda^{\dagger}\right) \otimes \zeta_{t}\left(\mu^{\dagger}, \lambda, \nu^{\dagger}\right) \\
& \longmapsto \sum_{v, i, j, r, t} F_{0, v}\left[\begin{array}{cc}
\mu^{\dagger} & \lambda^{\dagger} \\
\mu & \lambda
\end{array}\right]_{11}^{j i} R_{j r}^{2} R_{i t} F_{\mu^{\dagger}, 0}\left[\begin{array}{cc}
\lambda & \lambda^{\dagger} \\
v^{\dagger} & v
\end{array}\right]_{t r}^{11} \zeta\left(0, v^{\dagger}, v\right) \otimes \zeta\left(0, \lambda^{\dagger}, \lambda\right) \\
& =\sum_{\nu} E_{\lambda^{\dagger}}^{-1} N_{\mu, \nu}^{\lambda} \zeta\left(0, v^{\dagger}, v\right) \otimes \zeta\left(0, \lambda^{\dagger}, \lambda\right) \\
& \longmapsto \sum_{\nu} E_{\lambda^{\dagger}}^{-1} N_{\mu, \nu}^{\lambda} \zeta_{\nu}^{\alpha}
\end{aligned}
$$


Hence

$$
Z(\beta, \lambda)\left(\zeta_{\mu}^{\alpha}\right)=\sum_{v} N_{\mu, \nu}^{\lambda} \zeta_{v}^{\alpha}
$$

since

$$
\alpha_{\lambda}\left(Z(B) \zeta\left(\lambda, \lambda^{\dagger}, 0\right)\right)=E_{\lambda \dagger}
$$

by definition and by Lemma 7.2.

Proposition 7.6. We have the following equation:

$$
C_{\lambda, \mu}=S_{\mu, \lambda} / S_{\mu, 0}
$$

Proof. From the equation in Proposition 7.5 and (8), we deduce that

$$
S_{\mu, \varrho} C_{\lambda, \mu}=\sum_{v} N_{\nu, \varrho}^{\lambda} S_{\mu, \nu}
$$

Letting $\varrho=0$, we get that

$$
S_{\mu, 0} C_{\lambda, \mu}=S_{\mu, \lambda}
$$

which proves the stated equation, since we also conclude that $S_{\mu, 0} \neq 0$ from this.

Corollary 7.7. We have the following equation for the coefficients $c_{\kappa}$ :

$$
c_{\kappa}=d_{\kappa^{\dagger}} S_{\kappa^{\dagger}, 0} .
$$

Proof. One easily checks this equation by substitution.

\section{The formula for $S(\lambda)$}

We begin by establishing a formula for the curve operator in terms of the $F, R, B$, and the $d_{\mu}$ 's.

Lemma 8.1. For all $\kappa, \lambda \in \Lambda$ and all $j=1, \ldots N_{\kappa, v}^{v}$ we have that

$$
\begin{aligned}
& Z(\beta, \kappa)\left(\zeta_{i}\left(\lambda, \mu, \mu^{\dagger}\right)\right) \\
& \quad=\sum_{\nu, j, r, k, m, s, w, p} d_{v}^{-1} R_{i p} B_{p r} F_{v^{\dagger}, \mu}\left[\begin{array}{cc}
\kappa & \mu^{\dagger} \\
v^{\dagger} & \lambda
\end{array}\right]_{k r}^{s m} R_{m k} R_{s w}^{2} B_{w j} \zeta_{j}\left(\kappa, v, v^{\dagger}\right) .
\end{aligned}
$$

Proof. The curve operator $Z(\beta, \lambda)$ acts as follows (see Figure 9): 


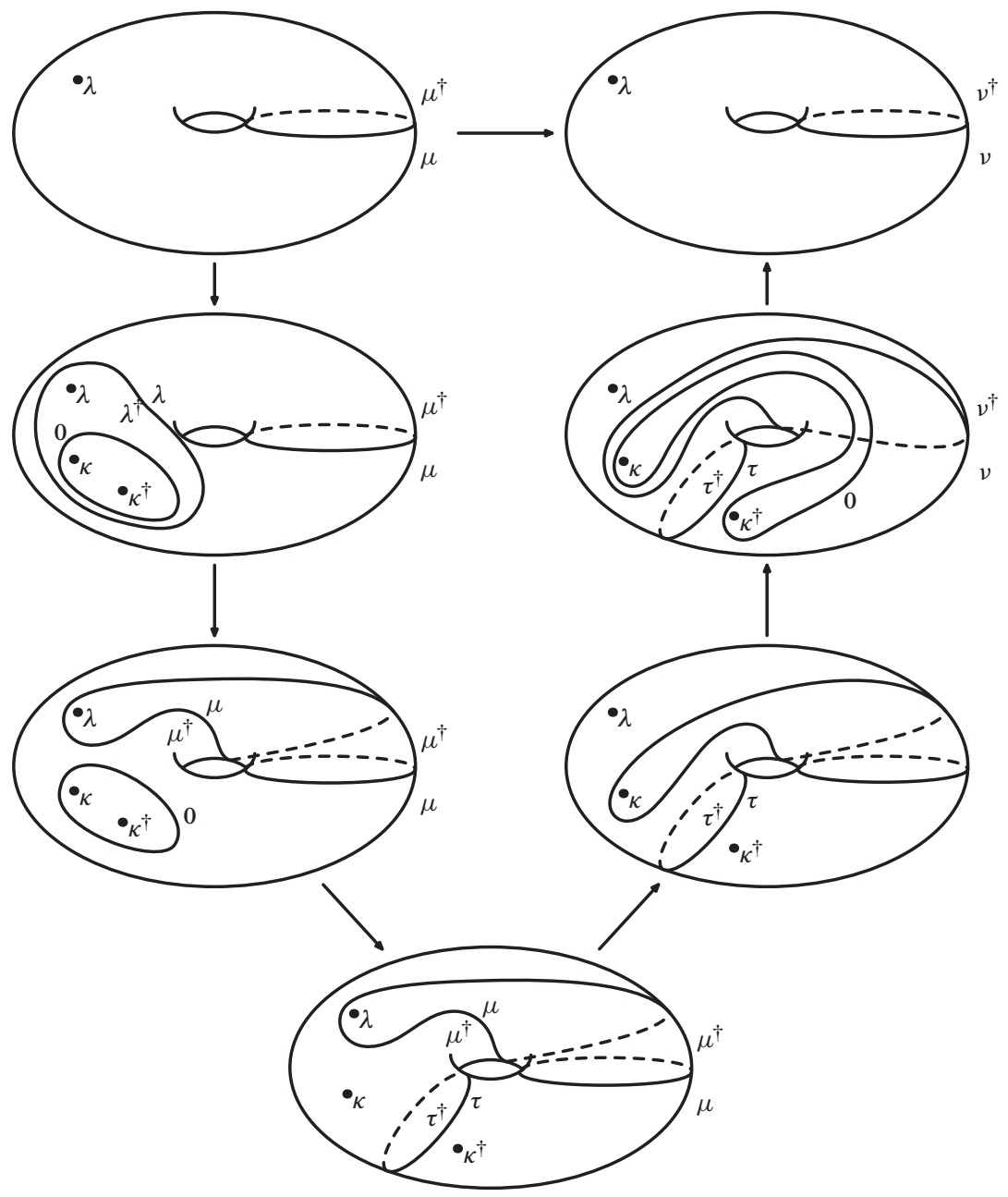

Figure 9. The curve operator along $\beta$ via changes $\mathcal{M}$ to $\mathcal{F}$.

$\zeta_{i}\left(\lambda, \mu, \mu^{\dagger}\right)$

$\zeta\left(\lambda^{\dagger}, \lambda, 0\right) \otimes \zeta_{i}\left(\lambda, \mu, \mu^{\dagger}\right) \otimes \zeta\left(0, \kappa, \kappa^{\dagger}\right)$

$\stackrel{(\mathrm{Id} \otimes R \otimes \mathrm{Id}) P^{231}(F \otimes \mathrm{Id})}{\longrightarrow} \sum_{p} R_{i p} \zeta\left(0, \kappa, \kappa^{\dagger}\right) \otimes \zeta\left(0, \mu, \mu^{\dagger}\right) \otimes \zeta_{p}\left(\mu, \mu^{\dagger}, \lambda\right)$

$\longmapsto \sum_{\tau, p, l, m} R_{i p} F_{0, \tau}\left[\begin{array}{cc}\kappa & \mu^{\dagger} \\ \kappa^{\dagger} & \mu\end{array}\right]_{11}^{l m}$

$$
\zeta_{l}\left(\tau, \kappa^{\dagger}, \mu\right) \otimes \zeta_{m}\left(\tau^{\dagger}, \mu^{\dagger}, \kappa\right) \otimes \zeta_{p}\left(\mu, \mu^{\dagger}, \lambda\right)
$$




$$
\begin{aligned}
& \longmapsto \sum_{\tau, p, k, l, m, r} R_{i p}\left(B_{23}\right)_{p r} R_{m k} F_{0, \tau}\left[\begin{array}{cc}
\kappa & \mu^{\dagger} \\
\kappa^{\dagger} & \mu
\end{array}\right]_{11}^{l m} \\
& \zeta_{l}\left(\tau, \kappa^{\dagger}, \mu\right) \otimes \zeta_{k}\left(\mu^{\dagger}, \kappa, \tau^{\dagger}\right) \otimes \zeta_{r}\left(\mu, \lambda, \mu^{\dagger}\right) \\
& \longmapsto \sum_{\tau, \nu, l, m, p, k, r, s, t}^{\mathrm{Id} \otimes F} R_{i p}\left(B_{23}\right)_{p r} R_{m k} F_{0, \tau}\left[\begin{array}{cc}
\kappa & \mu^{\dagger} \\
\kappa^{\dagger} & \mu
\end{array}\right]_{11}^{l m} F_{\mu \dagger, \nu}\left[\begin{array}{cc}
\kappa & \mu^{\dagger} \\
\tau^{\dagger} & \lambda
\end{array}\right]_{k r}^{s t} \\
& \zeta_{l}\left(\tau, \kappa^{\dagger}, \mu\right) \otimes \zeta_{s}\left(\nu, \tau^{\dagger}, \lambda\right) \otimes \zeta_{t}\left(v^{\dagger}, \mu^{\dagger}, \kappa\right) \\
& \stackrel{P^{231}\left(R^{2} \otimes \operatorname{Id} \otimes R\right)}{\longrightarrow} \sum_{\tau, v, l, m, p, k, r, s, t, u, v} R_{i p}\left(B_{23}\right)_{p r} R_{m k} F_{0, \tau}\left[\begin{array}{cc}
\kappa & \mu^{\dagger} \\
\kappa^{\dagger} & \mu
\end{array}\right]_{11}^{l m} F_{\mu \dagger, \nu}\left[\begin{array}{cc}
\kappa & \mu^{\dagger} \\
\tau^{\dagger} & \lambda
\end{array}\right]_{k r}^{s t} \\
& R_{l u}^{2} R_{t v} \zeta_{s}\left(v, \tau^{\dagger}, \lambda\right) \otimes \zeta_{v}\left(\mu^{\dagger}, \kappa, v^{\dagger}\right) \otimes \zeta_{u}\left(\mu, \tau, \kappa^{\dagger}\right) \\
& \longmapsto \sum_{\nu, l, m, p, k, r, s, t, u, v} R_{i p}\left(B_{23}\right)_{p r} R_{m k} F_{0, \nu}\left[\begin{array}{cc}
\kappa & \mu^{\dagger} \\
\kappa^{\dagger} & \mu
\end{array}\right]_{11}^{l m} F_{\mu \dagger, \nu}\left[\begin{array}{cc}
\kappa & \mu^{\dagger} \\
v^{\dagger} & \lambda
\end{array}\right]_{k r}^{s t} \\
& R_{l u}^{2} R_{t v} F_{\mu^{\dagger}, 0}\left[\begin{array}{cc}
\kappa & \kappa^{\dagger} \\
v^{\dagger} & v
\end{array}\right]_{v u}^{11} \zeta_{s}\left(v, v^{\dagger}, \lambda\right) \otimes \zeta\left(0, v^{\dagger}, v\right) \otimes \zeta\left(0, \kappa^{\dagger}, \kappa\right) .
\end{aligned}
$$

We now apply (6) to this expression, and we get that

$$
\begin{aligned}
& \zeta_{i}\left(\lambda, \mu, \mu^{\dagger}\right) \\
& \longmapsto \sum_{\nu, l, m, p, k, r, s} R_{i p}\left(B_{23}\right)_{p r} F_{\mu \dagger, v}\left[\begin{array}{cc}
\kappa & \mu^{\dagger} \\
v^{\dagger} & \lambda
\end{array}\right]_{k r}^{s m} R_{m k} \\
& \zeta_{s}\left(v, v^{\dagger}, \lambda\right) \otimes \zeta\left(0, v^{\dagger}, v\right) \otimes \zeta\left(0, \kappa^{\dagger}, \kappa\right) \\
& \longmapsto \sum_{\nu, l, m, p, k, r, s} R_{i p}\left(B_{23}\right)_{p r} F_{\mu \dagger, v}\left[\begin{array}{cc}
\kappa & \mu^{\dagger} \\
v^{\dagger} & \lambda
\end{array}\right]_{k r}^{s m} R_{m k}\left(B_{12}\right)_{s w} R_{w j}^{2} d_{\mu}^{-1} \zeta_{j}\left(\lambda, v, v^{\dagger}\right)
\end{aligned}
$$

from which the formula follows.

Proof of Theorem 1.2. By the calculations in Section 6 and Proposition 5.1, we get that

$$
S(\lambda)_{\mu, i}^{\nu, j}=\varepsilon d_{\nu} \sum_{\kappa} c_{\kappa} Z(\beta, \kappa)_{\mu, i}^{\nu, j} d_{\mu} .
$$

Combining this with (9) and the formula for $Z(\beta, \kappa)$ from Proposition 8.1, we obtain the equation stated in Theorem 1.2. 


\section{References}

[1] J.E. Andersen, Asymptotic faithfulness of the quantum SU( $n$ ) representations of the mapping class groups. Ann. of Math. (2) 163 (2006), 347-368. MR 2195137 Zbl 1157.53049 annals.math.princeton.edu/2006/163-1/p07

[2] J. E. Andersen, The Nielsen-Thurston classification of mapping classes is determined by TQFT. J. Math. Kyoto Univ. 48 (2008), 323-338. MR 2436739 Zbl 1195.57065

[3] J. E. Andersen, Asymptotics of the Hilbert-Schmidt norm in TQFT. Lett. Math. Phys. 91 (2010), 205-214. MR 2595923 Zbl 1195.57066

[4] J. E. Andersen, Toeplitz operators and Hitchin's projectively flat connection. In O. GarcíaPrada et al. (eds.), The many facets of geometry. A tribute to Nigel Hitchin. Oxford University Press, Oxford, 2010, 177-209. MR 2681692 Zbl 1213.53108

[5] J. E. Andersen, The Witten-Reshetikhin-Turaev invariants of finite order mapping tori I. To appear in J. Reine Angew. Math. Aarhus University Preprint 1995. arXiv:1104.5576v1

[6] J. E. Andersen, Mapping class groups do not have Kazhdan's Property (T). Preprint 2007 arXiv:0706.2184v1

[7] J. E. Andersen and J. L. Blaavand, Asymptotics of Toeplitz operators and applications in TQFT. In M. Schlichenmaier et al., Geometry and quantization. Lectures presented at the $3^{\text {rd }}$ international school and conference, Geoquant, Luxembourg City, Luxembourg, August 31-September 5, 2009. Travaux mathématiques 19. University of Luxembourg, Luxembourg, 2009, 167-201. MR 2883417 Zbl 1232.53077

[8] J.E. Andersen and N. L. Gammelgaard. Hitchin's projectively flat connection, Toeplitz operators and the asymptotic expansion of TQFT curve operators. In D. A. David and E. Previato (eds.), Grassmannians, moduli spaces and vector bundles Clay Mathematics Institute workshop on moduli spaces of vector bundles, with a view towards coherent sheaves, Cambridge (MA), U.S.A., October 6-11, 2006. Clay Mathematics Proceedings 14. Amer. Math. Soc., Providence (RI), and Clay Mathematics Institute, Cambridge (MA), 2011, 1-24. MR 2807846 Zbl 1232.53078

[9] J. E. Andersen and B. Himpel, The Witten-Reshetikhin-Turaev invariant of finite order mapping tori II. Quantum Topol. 3 (2012), 377-421.

[10] J. E. Andersen, G. Masbaum, and K. Ueno, Topological quantum field theory and the Nielsen-Thurston classification of M(0,4). Math. Proc. Cambridge Philos. Soc. 141 (206), 477-488. MR 2281410 Zbl 1110.57009

[11] J. E. Andersen and K. Ueno, Abelian conformal field theory and determinant bundles. Internat. J. Math. 18 (2007), 919-993. MR 2339577 Zbl 1128.81026

[12] J. E. Andersen and K. Ueno, Geometric construction of modular functors from conformal field theory. J. Knot Theory Ramifications 16 (2007), 127-202. MR 2306213 Zbl 1123.81041

[13] J. E. Andersen and K. Ueno, Construction of the Reshetikhin-Turaev TQFT from conformal field theory. Preprint 2011. arXiv:1110.5027v1

[14] M. F. Atiyah, On framings of 3-manifolds. Topology 29 (1990), 1-7. MR 1046621 Zbl 0716.57011 
[15] B. Bakalov and A. Jr. Kirillov, Lectures on tensor categories and modular functors. University Lecture Series 21. Amer. Math. Soc., Providence (RI), 2001. MR 1797619 Zbl 0965.18002

[16] C. Blanchet, Hecke algebras, modular categories and 3-manifolds quantum invariants. Topology 39 (2000), 193-223. MR 1710999 Zbl 0938.57009

[17] C. Blanchet, N. Habegger, G. Masbaum, and P. Vogel, Three-manifold invariants derived from the Kauffman bracket. Topology 31 (1992), 685-699. MR 1191373 Zbl 0771.57004

[18] C. Blanchet, N. Habegger, G. Masbaum, and P. Vogel, Topological quantum field theories derived from the Kauffman bracket. Topology 34 (1995), 883-927. MR 1362791 Zbl 0887.57009

[19] R. Dijkgraaf and E. Verlinde, Modular invariance and the fusion algebra. Nucl. Phys. B Proc. Suppl. 5B (1988), 87-97. MR 1002959 Zbl 0958.81510

[20] D. Friedan and S. Shenker, The analytic geometry of two-dimensional conformal field theory. Nucl. Phys. B 281 (1987), 509-545. MR 0869564

[21] J. Grove, Constructing TQFTs from modular functors. J. Knot Theory Ramifications 10 (2001), 1085-1131. MR 1871220 Zbl 1001.57050

[22] A. Hatcher and W. Thurston, A presentation for the mapping class group of a closed oriented surface. Topology 19, 221-237 (1980).

[23] M. Kontsevich, Rational conformal field theory and invariants of 3-manifolds. Preprint Centre de Physique Théorique Marseille 1988, CPT-88. www.ihes.fr/ $\sim$ maxim/TEXTS/3TQFT_and_RCFT.pdf

[24] G. Moore and N. Seiberg, Polynomial equations for rational conformal field theories. Phys. Lett. B 212 (1988), 451-460. MR 0962600

[25] G. Moore and N. Seiberg, Classical and quantum conformal field theory. Comm. Math. Phys. 123 (1989), 177-254. MR 1002038 Zbl 0694.53074

projecteuclid.org/getRecord?id=euclid.cmp/1104178762

[26] N. Yu. Reshetikhin and V. G. Turaev, Ribbon graphs and their invariants derived from quantum groups. Comm. Math. Phys. 127 (1990), 1-26. MR 1036112 Zbl 0768.57003 projecteuclid.org/getRecord?id=euclid.cmp/1104180037

[27] N. Yu. Reshetikhin and V. G. Turaev, Invariants of 3-manifolds via link polynomials and quantum groups. Invent. Math. 103 (1991), 547-597. MR 1091619 Zbl 0725.57007

[28] G. Segal, The definition of conformal field theory. In U. Tillmann (ed.), Topology, geometry and quantum field theory. Proceedings of the symposium in honour of the $60^{\text {th }}$ birthday of Graeme Segal held in Oxford, June 24-29, 2002. London Mathematical Society Lecture Note Series 308. Cambridge University Press, Cambridge, 2004. MR 2079383 Zbl 1076.19001

[29] A. Tsuchiya, K. Ueno, and Y. Yamada, Conformal Field Theory on Universal Family of Stable Curves with Gauge Symmetries. In M. Jimbo et al. (eds), Integrable systems in quantum field theory and statistical mechanics. Based on papers presented at the symposia held at Research Institute for Mathematical Sciences, Kyoto University, Kyoto (Japan) during October 17-21, 1988, and at Kyuzeso, Katata (Japan) during October 24-28, 1988. Advanced Studies in Pure Mathematics 19. Academic Press, Boston (MA), 1989, 459-566. MR 1048605 Zbl 0696.17010 
[30] V. G. Turaev, Quantum invariants of knots and 3-manifolds. De Gruyter Studies in Mathematics 18. Walter de Gruyter \& Co., Berlin, 1994. MR 1292673 Zbl 0812.57003

[31] K. Ueno, On conformal field theory. In N. J. Hitchin et al. (eds.), Vector bundles in algebraic geometry. Proceedings of the 1993 Durham symposium, Durham, U.K. Lond. Math. Soc. Lect. Note Ser. 208. Cambridge University Press, Cambridge, 1995, 283-345. MR 1338420 Zbl 0846.17027

[32] K. Ueno, Introduction to conformal field theory with gauge symmetries. In J. E. Andersen et al. (eds.), Geometry and physics. Proceedings of the conference at Aarhus University, Aarhus, Denmark, 1995. Lecture Notes in Pure and Appl. Math. 184. Marcel Dekker, New York, 1997, 603-745. MR 1423195 Zbl 0873.32022

[33] E. Verlinde, Fusion rules and modular transformations in 2D conformal field theory. Nucl. Phys. B 300 (1988), 360-376. MR 0954762 Zbl 1180.81120

[34] K. Walker, On Witten's 3-manifold invariants. Preprint 1991. Preliminary version \#2 tqft.net/other-papers/KevinWalkerTQFTNotes.pdf

[35] C. T. C. Wall, Non-additivity of the signature. Invent. Math. 7 (1969), 269-274. MR $0246311 \mathrm{Zbl} 0176.21501$

[36] E. Witten, Quantum field theory and the Jones polynomial. Comm. Math. Phys. 121 (1989), 351-399. MR 0990772 Zbl 0667.57005

projecteuclid.org/getRecord?id=euclid.cmp/1104178138

Received July 1, 2009

Jørgen Ellegaard Andersen, Center for Quantum Geometry of Moduli Spaces,

University of Aarhus, 8000 Aarhus, Denmark

E-mail: andersen@qgm.au.dk

Kenji Ueno, Department of Industrial and System Engineering,

Faculty of Science and Engineering, Hosei University, Koganei, Tokyo, 184-0002 Japan

E-mail: ueno@math.kyoto-u.ac.jp 